IZA DP No. 6667

Measuring Segregation When Hierarchy Matters

Robert Hutchens

June 2012 


\title{
Measuring Segregation When Hierarchy Matters
}

\author{
Robert Hutchens \\ Cornell University \\ and IZA
}

\section{Discussion Paper No. 6667 \\ June 2012}

\author{
IZA \\ P.O. Box 7240 \\ 53072 Bonn \\ Germany \\ Phone: +49-228-3894-0 \\ Fax: +49-228-3894-180 \\ E-mail: iza@iza.org
}

\begin{abstract}
Any opinions expressed here are those of the author(s) and not those of IZA. Research published in this series may include views on policy, but the institute itself takes no institutional policy positions.

The Institute for the Study of Labor (IZA) in Bonn is a local and virtual international research center and a place of communication between science, politics and business. IZA is an independent nonprofit organization supported by Deutsche Post Foundation. The center is associated with the University of Bonn and offers a stimulating research environment through its international network, workshops and conferences, data service, project support, research visits and doctoral program. IZA engages in (i) original and internationally competitive research in all fields of labor economics, (ii) development of policy concepts, and (iii) dissemination of research results and concepts to the interested public.
\end{abstract}

IZA Discussion Papers often represent preliminary work and are circulated to encourage discussion. Citation of such a paper should account for its provisional character. A revised version may be available directly from the author. 


\section{ABSTRACT}

\section{Measuring Segregation When Hierarchy Matters}

This paper considers the problem of measuring segregation when groups form a hierarchy whereby some groups have greater economic status than others. While existing measures of segregation address the case where people are unequally distributed across groups with the same economic status, concern often focuses on groups with different status, e.g., occupational segregation where women have limited access to high wage occupations. This paper first defines a class of segregation indexes that encompasses both the "same economic status" and "different economic status" case. It then proposes two methods for incorporating economic status into empirical work. One is to rank groups from highest to lowest economic status and apply the dominance criteria in Theorem 2. The other is to invoke a cardinal measure of group economic status and then compute a numerical index. Finally, a numerical index of segregation is introduced, and both methods are used to analyze U.S. occupational segregation by gender and ethnicity.

JEL Classification: $\quad$ C43, C81, D63, J15

Keywords: inequality, segregation, occupational segregation, inequality index, Lorenz dominance

Corresponding author:

Robert Hutchens

259 Ives Hall

Department of Economics

Cornell University

Ithaca, New York 14850

USA

E-mail: rmh2@cornell.edu 


\section{Measuring Segregation When Hierarchy Matters}

Measurement of segregation continues to be a topic of debate among economists and sociologists. This is in part because the underlying issues continue to be important. Women and minorities are underrepresented in some occupations, and there is reason to believe that unequal opportunity contributes to that. Similarly, some high schools have higher proportions of minorities than others, and that can both reflect and influence the quality of education at those schools. Measures of segregation help us examine such issues. ${ }^{1}$

This paper focuses on occupational segregation. Although the main ideas are applicable to other forms of segregation (e.g., housing or school segregation), occupational segregation provides a useful anchor for the exposition. Existing measures of occupational segregation compare two distributions of people across occupations in order to declare one distribution more segregated than another. These measures have a curious feature: they ignore the economic advantage of an occupation. That fits neither common parlance nor common practice. While separation of men and women into different occupations may be of concern, it is of particular concern when one group thereby suffers an economic disadvantage.

For example, in 1991 the U.S. Congress established the "Glass Ceiling Commission" to study barriers to the advancement of minorities and women within corporate hierarchies. The commission focused on executive occupations, noting that only $5 \%$ of senior managers in the Fortune 2000 companies are women (Federal Glass Ceiling Commission, p. 9). Of course, there are other occupations where women are similarly underrepresented (e.g., installation, maintenance, and repair). Congress presumably focused on the well-paid executive occupations not only because 
women and minorities were underrepresented in such occupations, but also because this underrepresentation was perceived to be accompanied by economic disadvantage. A good measure of segregation should arguably reflect such perceptions.

A similar issue comes up in discussions of role models. It is argued that the small numbers of women and minorities in high prestige occupations (e.g., scientist, astronaut, prime minister), affect the aspirations of the young and thereby investment in human capital. By implication, such segregation is particularly serious because it affects skill acquisition and thereby productivity. A good measure of segregation should arguably indicate that increased segregation in some occupations is of particular importance.

The goal of this paper is to develop and empirically implement measures of segregation that indicate the extent of segregation when occupations form a hierarchy. Section II defines a class of measures of segregation in hierarchies based on a cardinal scale of economic status. Measures of segregation within this class can indicate whether one distribution of people across occupations is less segregated -- in a sense that is made concrete below -- than another distribution. The class also includes measures where occupations have the same economic status, thereby permitting the kind of analysis performed with existing measures of segregation. For example, the square root measure of segregation in Hutchens (2001), which ignores economic status, is a member of the class. Section II closes with derivation of a unique measure of segregation in hierarchies (Corollary 1.1).

A problem with the Section II index is that it requires a cardinal scale of economic status, and existing scales differ in both derivation and outcome. ${ }^{2}$ Which scale is correct? The same problem arises when using equivalence scales to adjust income for family needs (e.g., numbers of children); there exist many equivalence scales, and no one is unambiguously superior. One branch of the literature resolves this problem by asserting that there may be no single correct cardinal equivalence 
scale, and then exploring less informationally demanding ordinal rankings of families, i.e., rankings from most needy family to least needy family.

Section III of this paper takes a similar approach. Suppose analysts disagree on the correct cardinal measure of economic status, but agree on the ordinal ranking of occupations from highest to lowest economic status. Given that ordinal ranking, are there conditions under which we can declare one occupational distribution of people more segregated than another? Theorem 2 gives an affirmative answer to this question. In doing so, it takes ideas from the literature on income inequality among heterogeneous agents (e.g., Atkinson and Bourguignon, 1987), ${ }^{3}$ and extends them to the problem of assessing occupational segregation among heterogeneous occupations.

The methods developed in Sections II and III are then applied in Section IV, which examines occupational segregation in the United States by gender and ethnicity. The results meaningfully differ from those obtained with existing measures of segregation.

\section{The Problem}

The first task is to introduce notation and frame the problem. Thus, consider a set of T groups that contain two types of people. Let $x_{i j}$ be the number of persons of type $\mathrm{i}$ in group $\mathrm{j}(\mathrm{i}=1,2$; $\mathrm{j}=1, \ldots, \mathrm{T})$. For example, type 1 could be women, type 2 could be men, and group $\mathrm{j}$ could be one of $\mathrm{T}$ occupations. The total number of people in group $\mathrm{j}$ is then equal to $x_{1 j}+x_{2 j}$. Let $x_{i}$ denote a row vector of numbers of type i people in occupations 1 through $\mathrm{T}$, i.e.,

$$
\begin{aligned}
& x_{1}=\left[x_{11}, x_{12}, \ldots, x_{1 \mathrm{~T}}\right] \\
& x_{2}=\left[x_{21}, x_{22}, \ldots, x_{2 \mathrm{~T}}\right] .
\end{aligned}
$$

Let $x$ be the matrix with $x=\left[\begin{array}{l}x_{1} \\ x_{2}\end{array}\right]$. For example, for four occupations, 


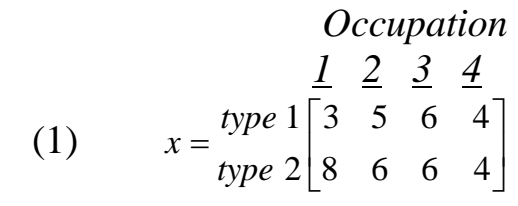

Denote the sum of type 1 and type 2 people as $N_{1}^{x}=\sum_{j} x_{1 j}$ and $N_{2}^{x}=\sum_{j} x_{2 j}$. Thus, if type 1 people are women, the distribution in (1) contains three women and eight men in the first occupation and a total of 18 women and 24 men $\left(N_{1}^{t}=3+5+6+4=18\right.$ and $\left.N_{2}^{t}=8+6+6+4=24\right)$.

The literature on measuring segregation seeks to assess whether one distribution of people across groups is more segregated (less equal) than another distribution. Thus, given an alternative distribution such as,

$$
y=\left[\begin{array}{llll}
1 & 4 & 5 & 1 \\
4 & 9 & 8 & 3
\end{array}\right]
$$

the fundamental question is whether $x$ is more segregated than $y$.

This paper addresses a slightly different problem: whether $x$ is more segregated than $y$ when segregation of one group is considered to be more important than segregation of another. Although there may be many reasons why segregation of a group is important, the focus here is on "differences in economic status." The idea is that there are group characteristics - characteristics other than the gender or racial composition of a group - that influence our assessment of segregation's severity. "Economic status" is used as shorthand for those other characteristics. ${ }^{4}$

In order to assess segregation when groups differ in economic status, assume that a single fixed number represents economic status. Let $k_{j}$ be a non-negative scalar associated with group $\mathrm{j}$ that indicates the economic status of that group, and let $k$ denote the vector of these numbers for groups 1 through T, i.e., $k=\left\{k_{1}, k_{2}, \ldots, k_{\mathrm{T}}\right\}$ 
If $k_{j}>k_{i}$, then group $\mathrm{j}$ has higher economic status than group $\mathrm{i}$, and if $k_{j}=k_{i}$, the two groups have the same economic status. In addition, when comparing distributions $x$ and $y$, the vector $k$ is assumed to be fixed in the sense that occupation $\mathrm{j}$ in both distributions has economic status $k_{\mathrm{j}}$.

The existing literature provides several measures of segregation that implicitly set $k_{1}=k_{2}$ $=\ldots=k_{\mathrm{T}}$. Perhaps the most general measure takes the form of comparing segregation curves. ${ }^{5}$ Segregation curves are formed by plotting the cumulative fraction of type 1 people (on the vertical axis) against the cumulative fraction of type 2 people (on the horizontal axis), with fractions ranked from low to high values of the ratio $x_{1 \mathrm{j}} / x_{2 \mathrm{j}}$. If the segregation curve for $x$ lies everywhere below that for $y$, then the $x$ distribution is more segregated than the $y$ distribution. Like the Lorenz curves used to measure income inequality, segregation curves yield a partial ordering of distributions; when segregation curves cross, they yield no information about whether $x$ is more segregated than $y$.

Therein lays the advantage to numerical measures of segregation. Although numerical measures rest on more stringent assumptions (or value judgments) about the nature of segregation than segregation curves, they have the advantage of yielding a complete ordering of distributions. With numerical measures the analyst can always determine whether $x$ is more segregated than $y$. The dissimilarity index in (2) and the square root index in (3) are examples of numerical measures of segregation.

$$
\begin{aligned}
& \text { (2) } O_{d}(x)=\frac{1}{2} \sum_{j=1}^{T}\left|x_{1 j} / N_{1}^{x}-x_{2 j} / N_{2}^{x}\right| \\
& \text { (3) } O(x)=1-\sum_{j=1}^{T} \sqrt{\left[x_{1 j} / N_{1}^{x}\right]\left[x_{2 j} / N_{2}^{x}\right]}
\end{aligned}
$$

Because neither segregation curves, the dissimilarity index, nor the square root index utilize information on the economic status of different groups, they implicitly assume, $k_{1}=k_{2}=\ldots=k_{\mathrm{T}}$. 
Since that case plays an important role in what follows, denote a $k$ vector with $k_{1}=k_{2}=\ldots=k_{\mathrm{T}}$ as $k^{0}$. This paper seeks to measure segregation when $k$ does not necessarily equal $k^{0}$.

Thus, the question posed in this paper is, "Given $k$, under what circumstances is $x$ more segregated than $y$ ?" The goal is to characterize a class of numerical measures that exhibit appropriate properties, and then use those measures to analyze data on segregation. Let $O(x ; k)$ be a continuous real valued measure of segregation defined on the domain $D$. Denoting the vector space of all $2 \times \mathrm{T}$ real matrices with non-negative elements by $\mathbf{R}_{+}^{2 \times T}$, the domain of $O(x ; k)$ is $D=\bigcup_{T=2}^{\infty} D_{T}$ where $D_{T}=\left\{x \in \mathbf{R}_{+}^{2 \times T}\right.$ and $\left.k \in \mathbf{R}_{+}^{T}: N_{i}^{x}>0, i=1,2\right\} .{ }^{6}$ Since $O(x ; k)$ is a measure of segregation, $O(x ; k) \geq O(y ; k)$ iff $x$ is at least as segregated as $y$ for each $x, y$ and $k$ element of $D$. This paper thus seeks to characterize $O(x ; k) .^{7}$

\section{Properties and a Theorem}

We begin with the case where the elements of $k$ are cardinal measures of group economic status. Of course, this is problematic; it may be difficult to reach agreement on the "true" cardinal measure of economic status. That issue is dealt with in the Section III treatment of ordinal rankings. This section first states properties for the special case where $k=k^{0}$, then moves to properties for $k \neq$ $k^{0}$, and concludes with a theorem that links the properties to a specific measure of segregation. Since the existing literature stipulates desirable properties when $k=k^{0}$, it is important to start there. An index that is defined for all $k$ should exhibit those same desirable properties when $k=k^{0}$; if it violates the properties, then either the index or the existing literature is wrong.

Hutchens (2001) presents seven properties for a "good" numerical measure of segregation and establishes the class of measures for which these properties are necessary and sufficient. ${ }^{8}$ While 
a statement of these properties has the appearance of going over old ground, in fact, it is more than that. The properties specify the behavior of $O(x ; k)$ in the special case where $k=k^{0}$, and thereby serve to narrow the class of acceptable indexes. Moreover, since they belong to an earlier literature, the seven properties have the virtue of having been vetted as desirable for a measure of segregation. To state the properties, two definitions are required:

A Proportional Division. The distribution $y$ is obtained from $x$ by a proportional division of a group if $y_{i j}=x_{i j}, \mathrm{i}=1,2 ; \mathrm{j}=1, . ., \mathrm{T}-1$ $y_{i j}=x_{i \mathrm{~T}} / \mathrm{M}, \mathrm{i}=1,2 ; \mathrm{j}=\mathrm{T}, \ldots, \mathrm{T}+\mathrm{M}-1$, where $\mathrm{M}$ is a positive integer.

A Disequalizing Movement. The distribution $y$ is obtained from $x$ by a disequalizing movement of type 1 people if, for $\mathrm{f}$ and g, (a) $x_{2 f}=x_{2 g}=y_{2 f}=y_{2 g}>0$, (b) $x_{1 f} / x_{2 f}<x_{1 g} / x_{2 g}$, (c) $y_{1 f}=x_{1 f}-d$ and $y_{1 g}=x_{1 g}+d$, for $0<d \leq x_{1 f}$, and (d) $x_{i h}=y_{i h}, \mathrm{i}=1,2 ; h \neq \mathrm{f}, \mathrm{g}$.

A proportional division divides a group into $\mathrm{M}$ subgroups, such that each of the subgroups contain $1 / \mathrm{M}$ of the original type 1 and type 2 people. As a result, the ratio of type 1 to type 2 people in each of the subgroups is the same as the original (pre-division) ratio. $\mathrm{P} 2$ below addresses the effect of a proportional division on measured segregation. It essentially says that a segregation index should not change as a result of a proportional division.

A disequalizing movement is similar to a regressive transfer in the income inequality literature. Given two groups, f and g, with $x_{1 f} / x_{2 f}<x_{1 g} / x_{2 g}$, a disequalizing movement reduces $x_{1 f} / x_{2 f}$ and increases $x_{1 g} / x_{2 g}$. To illustrate, let type 1 people be women and type 2 people be men, and suppose that the initial distribution is as in (1) above. Note that since occupation 2 contains 5 women and 6 men and occupation 3 contains 6 women and 6 men, $x_{12} / x_{22}=5 / 6<6 / 6=x_{13} / x_{23}$. Now, suppose " $d$ " women move from occupation 2 to occupation 3 (so that occupation 2 contains 5- $d$ women and occupation 3 contains $6+d$ women), where $d$ is small. This movement increases the female to male ratio in the more "female" occupation 3 while decreasing that ratio in the more 
"male" occupation 2. P7 below addresses the effect of such a disequalizing movement; it essentially says that measured segregation should either increase or not change as a result.

Given these definitions, the seven properties in Hutchens (2001) can be written,

P1. Scale Invariance. Let $\Phi=\left[\begin{array}{cc}\alpha & 0 \\ 0 & \beta\end{array}\right]$ where $\alpha$ and $\beta$ are positive scalars. If $y=\Phi x$, then $O\left(x ; k^{0}\right)=O\left(y ; k^{0}\right)$.

P2. Insensitivity to Proportional Divisions. If $y$ is obtained from $x$ by a proportional division of a group, then $O\left(x ; k^{0}\right)=O\left(y ; k^{0}\right)$.

P3. Zero Member Independence. If $x$ and $y$ are identical except that $x$ includes a group with no members and $y$ excludes that group, then $O\left(x ; k^{0}\right)=O\left(y ; k^{0}\right)$.

P4. Symmetry in Types. If type 1 and type 2 people exchange labels such that $x=\left[\begin{array}{l}x_{1} \\ x_{2}\end{array}\right]$ and $y=\left[\begin{array}{l}x_{2} \\ x_{1}\end{array}\right]$ then $O\left(x ; k^{0}\right)=O\left(y ; k^{0}\right)$.

P5. Additivity. $O\left(x ; k^{0}\right)$ is additive in groups, i.e., $O\left(x ; k^{0}\right)=\Gamma\left\{\sum_{j=1}^{T} O^{j}\left(x_{1 j}, x_{2 j} ; k_{j}^{0}\right)\right\}$ where $O^{l}(), O^{2}(), \ldots, O^{T}()$ are continuous real-valued functions from $\mathbf{R}_{+}^{2} \times \mathbf{R}_{+} \rightarrow \mathbf{R}$, and $\Gamma\{\}$ is continuous and strictly increasing.

P6. Symmetry In Groups. If $\left(\mathrm{j}_{1}, \ldots, \mathrm{j}_{\mathrm{T}}\right)$ is any permutation of $1, \ldots, \mathrm{T}$, $x=\left[\begin{array}{c}x_{11}, x_{12}, \cdots, x_{1 T} \\ x_{21}, x_{22}, \cdots, x_{2 T}\end{array}\right]$, and $y=\left[\begin{array}{c}x_{1 j_{1}}, x_{1 j_{2}}, \cdots, x_{1 j_{T}} \\ x_{2 j_{1}}, x_{2 j_{2}}, \cdots, x_{2 j_{T}}\end{array}\right]$ then $O\left(x ; k^{0}\right)=O\left(y ; k^{0}\right)$.

P7. Movement Between Groups. If $y$ is obtained from $x$ by a disequalizing movement, then $O\left(x ; k^{0}\right) \leq O\left(y ; k^{0}\right) .^{9}$

Additional explanation may be helpful here. P1 simply says that in the case where $k=k^{0}$, if $N_{1}$ is multiplied by a positive scalar $(\alpha)$ and the shares of type 1 people in each occupation do not change (where a share is calculated as the number of type 1 people in an occupation divided by $N_{1}$ ), then segregation does not change. To illustrate, let type 1 be women, and assume the number of women in the labor force doubles $(\alpha=2)$. If the occupations that previously contained $0 \%, 10 \%, 20 \%$, etc. of all women continue to do so, then this change in the level of women in the population does not affect 
segregation. As noted above, $\mathrm{P} 2$ says that a proportional division should not change measured segregation. A proportional division essentially allocates the old segregation over new occupations; the ratio of type 1 to type 2 people in the new occupations is the same as in the old. P3 - P6 are selfexplanatory. As noted above, P7 is similar to the principle of transfers in the income inequality literature. The principle of transfers says that a transfer of income from a low income person to a high income person can not decrease and may increase income inequality; P7 says that a movement of type 1 people from a group where the ratio of type 1 to type 2 is low into a group where that ratio is high can not decrease and may increase segregation.

Note that P7 focuses on type 1 people. One could posit a similar property for type 2 people. Thanks in part to symmetry in types (P4), the subsequent theorems can be derived without a parallel property for type 2 people. Thus, in keeping with properties that are as unrestrictive as possible, it is sufficient to focus on type 1 people. Of course, who is labeled type 1 or type 2 is arbitrary. The same point applies to $\mathrm{P} 7$ ' and $\mathrm{P} 8$ ' below.

In the special case where $k=k^{0}$, the index in this paper should satisfy the above seven properties. The index must, however, also address cases where $k \neq k^{0}$. Four additional properties -properties that extend properties 5-7 above to cases where $k \neq k^{0}--$ are useful. First, it is necessary to modify the functional form of the index and to redefine symmetry. Thus,

P5'. Additivity For All $k . O(x ; k)$ is additive in groups, i.e., $O(x ; k)=\Gamma\left\{\sum_{j=1}^{T} O^{j}\left(x_{1 j}, x_{2 j} ; k_{j}\right)\right\}$ where $O^{1}\left(\right.$ ), $O^{2}\left(\right.$ ), ..., $O^{T}\left(\right.$ ) are 3-smooth real-valued functions from $\mathbf{R}_{+}^{2} \times \mathbf{R}_{+} \rightarrow \mathbf{R}$, and $\Gamma\{\}$ is differentiable and strictly increasing.

Additivity is a reasonable property because it is reasonable to assert that total segregation can be decomposed into components that are attributable to subsets of groups. For example, one might seek 
to determine what fraction of segregation for all occupations arises in blue-collar occupations. Such an exercise can only be meaningful if segregation in the blue-collar subset is not affected by changes in segregation outside of that subset. Only then can total segregation be split into distinct "within blue-collar" and "not within blue-collar" categories. Like P5, P5' is sufficient to insure that that is true for the blue-collar subset, the white-collar subset, or any other feasible partition of the occupations. ${ }^{10}$ Unlike P5, P5' deals with $k \neq k^{0}$, and requires that the function $O($ ) be 3 -smooth (thus, the function and its first, second, and third derivatives are continuous).

This is a good place to introduce a second, more stringent, assumption about functional form: additive decomposability. As established below, this assumption leads to a unique segregation index. Introducing notation, partition the $x$ matrix into $\mathrm{G}$ mutually exclusive and exhaustive subsets containing $\mathrm{T}^{1}, \ldots, \mathrm{T}^{\mathrm{G}}$ occupations such that $x=\left\{x^{1}, \ldots, x^{\mathrm{G}}\right\}$ where $x^{\mathrm{g}}$ is a $2 \times \mathrm{T}^{\mathrm{g}}$ matrix that denotes the distribution of type 1 and type 2 people across the $\mathrm{T}^{\mathrm{g}}$ occupations, and $\mathrm{T}=\Sigma_{\mathrm{g}} \mathrm{T}^{\mathrm{g}}$. Then proceeding in a manner similar to Hutchens (2004),

Additive Decomposability. A measure of segregation is additive decomposable for groups with the same economic status if it takes the form,

$$
O\left(x ; k^{0}\right)=\sum_{g=1}^{G} w_{g} O\left(x^{g} ; k^{0}\right)+B
$$

where $\mathrm{w}_{\mathrm{g}}$ is the weight attached to subset $\mathrm{g}(\mathrm{g}=1, \ldots, \mathrm{G}), \mathrm{B}$ is the between subset term, and

$$
\begin{aligned}
& \mathrm{w}_{\mathrm{g}}=\mathrm{w}_{\mathrm{g}}\left(\mathrm{N}_{1}\left(x^{1}\right), \ldots, \mathrm{N}_{1}\left(x^{\mathrm{G}}\right), \mathrm{N}_{2}\left(x^{1}\right), \ldots, \mathrm{N}_{2}\left(x^{\mathrm{G}}\right), k^{0}\right)>0 \\
& \mathrm{~B}=\mathrm{B}\left(\mathrm{N}_{1}\left(x^{1}\right), \ldots, \mathrm{N}_{1}\left(x^{\mathrm{G}}\right), \mathrm{N}_{2}\left(x^{1}\right), \ldots, \mathrm{N}_{2}\left(x^{\mathrm{G}}\right), k^{0}\right) .
\end{aligned}
$$

Additive decomposable measures of inequality are useful because they permit a decomposition of inequality into "within" and "between" components. For example, an additive decomposable measure of segregation permits a decomposition of total segregation into a within-blue collar component, a within-white collar component, and a between white and blue collar component. For 
present purposes, a further advantage of additive decomposability is that it can be used to restrict P5'

in a way that yields interesting results. Thus, we consider the following restriction on P5',

P5'. Extended Additivity. $O(x ; k)$ is not only additive for all $k(\mathrm{P} 5$ '), but also additive decomposable for groups with the same economic status.

The next property, P6', simply extends symmetry in groups (P6) to cases where the elements of $k$ differ. Thus, if occupations are relabeled, so that occupation 3 is labeled 4 and occupation 4 is labeled 3, then segregation is unaffected; the occupations have the same value of $k$ and the same count of men and women as before; only the labels changed.

P6'. Symmetry In Groups with Different Values of $\boldsymbol{k}$. Assume that $\left(\mathrm{j}_{1}, \ldots, \mathrm{j}_{\mathrm{T}}\right)$ is any permutation of $1, \ldots, \mathrm{T}$, and let $x=\left[\begin{array}{c}x_{11}, x_{12}, \cdots, x_{1 T} \\ x_{21}, x_{22}, \cdots, x_{2 T}\end{array}\right], y=\left[\begin{array}{c}x_{1 j_{1}}, x_{1 j_{2}}, \cdots, x_{1 j_{T}} \\ x_{2 j_{1}}, x_{2 j_{2}}, \cdots, x_{2 j_{T}}\end{array}\right], k=\left(k_{1}, k_{2}, \ldots, k_{T}\right)$, and $k^{\prime}=\left(k_{j_{1}}, k_{j_{2}}, \ldots, k_{j_{T}}\right)$.

Then $O(x ; k)=O\left(y ; k^{\prime}\right)$.

The next two properties -- P7' and P8' -- address movements between groups when groups have different levels of economic status. In discussing these properties, it is useful to introduce a distinction between two types of disequalizing movements: "a disequalizing movement with reduced economic status" and "a disequalizing movement with unchanged economic status." The first is simply a disequalizing movement between two groups such that the "receiving" group has less economic status than the "sending" group. Thus, adding part (e) to the previous definition, A Disequalizing Movement with Reduced Economic Status. The distribution $y$ is obtained from $x$ by a disequalizing movement with reduced economic status of type 1 people if, for $\mathrm{f}$ and $\mathrm{g}$, (a) $x_{2 f}=x_{2 g}=$ $y_{2 f}=y_{2 g}>0$, (b) $x_{1 f} / x_{2 f}<x_{1 g} / x_{2 g}$, (c) $y_{1 f}=x_{1 f}-d$ and $y_{1 g}=x_{1 g}+d$, for $0<d \leq x_{1 f}$, (d) $x_{i h}=y_{i h}, \mathrm{i}=1,2 ; h$ $\neq \mathrm{f}, \mathrm{g},(\mathrm{e}) k_{f}>k_{g}$.

A disequalizing movement with unchanged economic status is defined identically, except that part

(e) becomes $k_{f}=k_{g}{ }^{11}$ 
An illustration may be helpful here. Let type 1 people be women and type 2 people be men, and suppose that the initial $x$ distribution is as in (4), with $\mathrm{k}_{\mathrm{f}}=\mathrm{k}_{\mathrm{g}}>\mathrm{k}_{\mathrm{h}}$ by assumption.

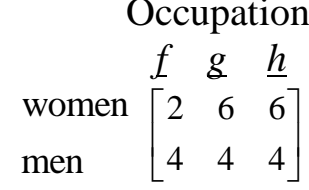

Now form the $y$ distribution by moving $d$ women from occupation $\mathrm{f}$ to occupation $\mathrm{g}$ (so that occupation f contains 2- $d$ women and 4 men and occupation g contains $6+d$ women and 4 men). Since $x_{1 f} / x_{2 f}<x_{1 g} / x_{2 g}$ and $k_{\mathrm{f}}=k_{\mathrm{g}}$, this is a disequalizing movement with unchanged economic status. In accordance with $\mathrm{P} 7$ such a disequalizing movement may increase segregation. Next, form the $z$ distribution by moving $d$ women from occupation $\mathrm{f}$ to occupation h. Since $x_{1 \mathrm{f}} / x_{2 f}<x_{1 h} / x_{2 h}$ and $k_{\mathrm{f}}>k_{\mathrm{h}}$, this is a disequalizing movement with reduced economic status. As stated in the following property, because people suffer reduced status, this movement from $\mathrm{f}$ to $\mathrm{h}$ can have a larger effect on segregation than the otherwise identical disequalizing movement with unchanged economic status (from $\mathrm{f}$ to $\mathrm{g}$ ). This is because the $d$ women who move from $\mathrm{f}$ to $\mathrm{h}$ suffer reduced economic status, while the $d$ women who move from $\mathrm{f}$ to $\mathrm{g}$ do not.

P7'. Movements Between Groups with Different Values of $\boldsymbol{k}$. Assume that the $x$ distribution has T groups. Denote three of these groups as f, g, and h with $x_{1 f}<x_{1 g}=x_{1 h}$ and $k_{f}=k_{g}>k_{h}$. With no change in $k$, let the $y$ distribution be obtained from $x$ through a movement of $d$ type 1 people from group $\mathrm{f}$ to group $\mathrm{g}$, and let the $z$ distribution be obtained from $x$ through a movement of $d$ type 1 people from group $\mathrm{f}$ to group $\mathrm{h}$. Then for all $0 \leq d \leq x_{1 f}$,

(a) if $x_{2 f}=x_{2 g}=x_{2 h}>0$, then $O(x ; k) \leq O(y ; k) \leq O(z ; k)$

(b) if $x_{2 f}=x_{2 g}=x_{2 h}=0$ then $O(x ; k)=O(y ; k) \leq O(z ; k)$

Statement (a) in P7' essentially makes two points. First, a disequalizing movement with unchanged economic status should not decrease and may increase segregation. This essentially repeats P7 above. Second, a disequalizing movement with reduced economic status should have an effect on measured segregation that exceeds or equals an otherwise identical disequalizing movement 
with unchanged economic status. Thus, increased segregation may be more egregious in some occupations than in others; while a disequalizing movement may increase segregation, it can increase segregation even more if the movers suffer reduced economic status.

Statement (b) in P7' addresses the effect on segregation of a movement of type 1 people between two groups that only contain type 1 people, i.e., the two groups are completely segregated. It essentially says that if the two groups have the same economic status, then the movement does not affect measured segregation. Nothing changed; the type 1 people were completely segregated and had the same economic status before and after the move. If the movement involves reduced economic status (the receiving group has lower economic status than the sending group), then the segregation index should not decrease and may increase. In other words, since the women suffer reduced economic status, an increase in measured segregation cannot be ruled out. ${ }^{12}$

The next property considers two pairs of groups: the first pair consists of groups $\mathrm{f}$ and $\mathrm{g}$, and the second of groups $q$ and $r$. The property addresses the effect of identical disequalizing movements within each pair when $k_{\mathrm{f}}=k_{g}<k_{q}=k_{r}$.

\section{P8'. Movements Between Groups with the Same Value of $k$.}

Assume that the $x$ distribution has T groups. Denote four of these groups as $\mathrm{f}, \mathrm{g}, \mathrm{q}$, and $\mathrm{r}$ with $0<x_{1 f}=x_{1 q}<x_{1 g}=x_{1 r}$ and $k_{f}=k_{g}<k_{q}=k_{r}$. With no change in $k$, let the $y$ distribution be obtained from $x$ through a movement of $d$ type 1 people from group $\mathrm{f}$ to group g, and let the $v$ distribution be obtained from $x$ through a movement of $d$ type 1 people from group q to group r. Then for all $0 \leq d \leq x_{1 f}$,

(a) if $x_{2 f}=x_{2 g}=x_{2 q}=x_{2 r}>0$ then $\mathrm{O}(x ; k) \leq \mathrm{O}(y ; k) \leq \mathrm{O}(v ; k)$

(b) if $x_{2 f}=x_{2 g}=x_{2 q}=x_{2 r}=0$ then $\mathrm{O}(x ; k)=\mathrm{O}(y ; k)=\mathrm{O}(v ; k)$

Statement (a) in P8' essentially says that a small disequalizing movement between two equally high economic status occupations should have an effect on measured segregation that is greater than or equal to an otherwise identical disequalizing movement between two equally low economic status occupations. Alternatively stated, the change in segregation resulting from a 
disequalizing movement between two occupations with the same value of $k$ should be non-decreasing in $k$. This is arguably consistent with the values underlying establishment of the 1991 Glass Ceiling Commission. Societies need to put special emphasis on reducing segregation in high economic status occupations. While increased occupational segregation is always worrisome, increased occupational segregation in high economic status occupations is particularly worrisome. ${ }^{13}$

Like P8'(a), statement P8'(b) considers a movement of type 1 people when $k_{\mathrm{f}}=k_{g}<k_{q}=k_{r}$. The only difference is that in statement (b) both pairs of groups are completely segregated, i.e., the groups contain only type 1 people. As in P7'(b), if two groups are of equal economic status and are completely segregated such that there are only type 1 people in the two groups, then movement of type 1 people between the two groups does not change measured segregation. The movement changes neither their economic status nor their degree of segregation.

Properties P5' - P8' in combination with P1 - P4 yield the following specification of what is henceforth termed a "status-augmented measure of segregation,"

Theorem 1. $O(x ; k)$ satisfies properties $1-4,5,-8$ ' if and only if

$$
O(x ; k)=\Gamma\left\{\sum_{j=1}^{T}\left[L\left(s_{1 j}, s_{2 j} ; k_{j}\right)+\lambda\left(k_{j}\right)\left(s_{1 j}+s_{2 j}\right)\right]\right\}, \text { where }
$$

$\Gamma\{\}: \mathbf{R} \rightarrow \mathbf{R}$ is differentiable and strictly increasing, $s_{i j}=x_{i j} / N_{i}^{x}, \mathrm{i}=1,2 ; \mathrm{j}=1, . ., \mathrm{T}$,

$\lambda\left(k_{j}\right): \mathbf{R}_{+} \rightarrow \mathbf{R}$ is a non-increasing differentiable function of $k_{j}$, and $L(): \mathbf{R}_{+}^{2} \times \mathbf{R}_{+} \rightarrow \mathbf{R}$ is a 3-smooth function with
a) $L\left(0,0 ; k_{j}\right)=L\left(s_{1 j}, 0 ; k_{j}\right)=L\left(0, s_{2 j} ; k_{j}\right)=0, \mathrm{j}=1, . ., \mathrm{T}$.
b) $L($ ) is symmetric, and homogeneous of degree one in its first two arguments, i.e., $L\left(s_{1 j}, s_{2 j} ; k_{j}\right)=L\left(s_{2 j}, s_{1 j} ; k_{j}\right)$ and $L\left(s_{1 j}, s_{2 j} ; k_{j}\right)=L\left(\delta s_{1 j}, \delta s_{2 j} ; k_{j}\right) / \delta$ for $\delta>0$.
c) $\partial^{2} L() / \partial s_{i j} \partial k_{j}+\partial \lambda\left(k_{j}\right) / \partial k_{j} \leq 0, \mathrm{i}=1,2 ; \mathrm{j}=1, \ldots, \mathrm{T}$.
d) $\partial^{3} L() / \partial s_{1 j}^{2} \partial k_{j} \geq 0$ if $s_{2 j}>0$ and $\partial^{3} L() / \partial s_{1 j}^{2} \partial k_{j}=0$ if $s_{2 j}=0, \mathrm{j}=1, . ., \mathrm{T}$.
e) $\partial^{2} L() / \partial s_{1 j}^{2} \geq 0$ if $s_{2 j}>0$ and $\partial^{2} L() / \partial s_{1 j}^{2}=0$ if $s_{2 j}=0, \mathrm{j}=1, . ., \mathrm{T}$
f) $\partial^{3} L() / \partial s_{2 j}^{2} \partial k_{j} \geq 0$ if $s_{1 j}>0$ and $\partial^{3} L() / \partial s_{2 j}^{2} \partial k_{j}=0$ if $s_{1 j}=0, \mathrm{j}=1, . ., \mathrm{T}$. 
g) $\partial^{2} L() / \partial s_{2 j}^{2} \geq 0$ if $s_{1 j}>0$ and $\partial^{2} L() / \partial s_{2 j}^{2}=0$ if $s_{1 j}=0, \mathrm{j}=1, . ., \mathrm{T}$

Proof: See Appendix.

As noted above, when P5' is replaced by P5', a unique measure emerges. Specifically,

Corollary 1.1: $O(x ; k)$ satisfies properties $1-4,6{ }^{\prime}-8$ ' as well as $\mathrm{P} 5$ ', if and only if it takes the form,

$$
O(x ; k)=O_{b}(x ; k)=b-\gamma(\tilde{k}) 2 \lambda(\tilde{k})+\gamma(\tilde{k}) \sum_{j=1}^{T}\left[\lambda\left(k_{j}\right)\left(s_{1 j}+s_{2 j}\right)-\frac{b}{\gamma\left(k_{j}\right)} \sqrt{s_{1 j} s_{2 j}}\right]
$$

where $b$ is positive, $\lambda\left(k_{\mathrm{j}}\right)$ is a nonincreasing differentiable function of $k_{\mathrm{j}}, \gamma\left(k_{j}\right)$ is positive, non -increasing and differentiable in $k_{\mathrm{j}}$, and $\tilde{k}$ is a non-negative number.

Proof: See Appendix

In the special case where $k=k^{0}$, one would expect the Theorem 1 index to also satisfy P1-P7.

The following corollary establishes that connection:

Corollary 1.2. If $k_{1}^{0}=k_{2}^{0}=\ldots=k_{T}^{0}$, then (5) satisfies properties 1-7.

Proof: See Appendix

Note that equation (6) provides an example of an index that meets the requirements of

Theorem 1. Theorem 1 specifies $L($ ) as a 3-smooth function that is symmetric, homogeneous of degree one in $s_{1 j}$ and $s_{2 j}$, and that equals zero when $s_{1 j}$ and/or $s_{2 j}$ equal zero. The function $L\left(s_{1 j}, s_{2 j} ; k_{j}\right)=-\frac{b}{\gamma\left(k_{j}\right)} \sqrt{s_{1 j} s_{2 j}}$ not only meets these requirements, but also possesses the derivatives stipulated in $(\mathrm{c})-(\mathrm{g})$ of Theorem $1 .^{14}$

Perhaps the most unusual feature of the indexes in (5) and (6) - at least from the perspective of existing measures of segregation like (2) and (3) - is the term, $\sum_{j=1}^{T}\left[\lambda\left(k_{j}\right)\left(s_{1 j}+s_{2 j}\right)\right]$. The term essentially arises out of P7'(b); it assures that in the case where $k_{\mathrm{f}}>k_{\mathrm{h}}$ and $s_{2 \mathrm{f}}=s_{2 \mathrm{~h}}=0$, a movement of $d$ type 1 people from high status group f to lower status group h cannot decrease and may increase measured segregation. ${ }^{15}$ 
For purposes of understanding Corollary 1.2, it is instructive to consider a variant of the index in equation (6). Let $\tilde{k}=k^{1}=k^{2}=\ldots=k^{T}$ and $\mathrm{b}=1$. Then (6) reduces to, (7) $\quad 1-\sum_{j}^{T} \sqrt{\left[s_{1 j}\right]\left[s_{2 j}\right]}$

which is identical to the square root index in (3) above. In accordance with Corollary 1.2, the index in equation (7) should satisfy properties $1-7$. Not only is that easily confirmed, it is consistent with Hutchens (2001), which proves that the square root index satisfies the seven properties. Given this connection, the equation (6) index is henceforth termed a "status-augmented square root index," and the equations (3) and (7) index termed a "status-free square root index."

In summary, from Theorem 1 and its corollaries we learn that the stated properties are quite restrictive about how one should incorporate economic status into a measure of segregation. When hierarchy matters, measurement of segregation requires a complex interaction between the measure of economic status $(k)$ and standard status-free measures of segregation.

\section{Assessing Segregation When There Is Only an Ordinal Ranking of the $k_{j}$}

While the augmented square root index in equation (6) has several desirable properties, it also has a weakness: it requires an acceptable cardinal measure of economic status. Consider, for example, an analysis of occupational segregation that uses average earnings as a measure of economic status. A critic might plausibly ask whether we really believe that an occupation with twice the earnings of another has exactly twice the economic status. Of course, this is a problem for any member of the class of measures encompassed by Theorem 1 .

An alternative way to proceed is to assume that while analysts may not agree on a cardinal measure of occupational economic status, they can still rank occupations from lowest to highest economic status. Thus, instead of claiming that occupation A has twice the economic status of B, one 
is willing to say that A has greater economic status than B. By this assumption, the only useful information in a measure of economic status is the ordinal ranking $k_{1} \geq \ldots \geq k_{\mathrm{T}}$. To make the point another way, consider two measures of economic status $k^{\prime}=\left\{k_{1}^{\prime}, k_{2}^{\prime}, \ldots, k_{T}^{\prime}\right\}$ and $k^{\prime \prime}=\left\{k_{1}^{\prime \prime}, k_{2}^{\prime \prime}, \ldots, k_{T}^{\prime \prime}\right\}$ with $k_{j}^{\prime} \neq k_{j}^{\prime \prime}, \mathrm{j}=1, \ldots, \mathrm{T}$; this section assumes that if $k_{1}^{\prime} \geq \ldots \geq k_{T}^{\prime} \geq 0$ and $k_{1}^{\prime \prime} \geq \ldots \geq k_{T}^{\prime \prime} \geq 0$, then $k^{\prime}$ and $k^{\prime \prime}$ convey identical information about economic status. In that case, is it still possible to make statements about occupational segregation? More precisely, with the T groups fixed in descending order of status, are there conditions on $x$ and $y$ that guarantee that $O(x ; k) \geq O(y ; k)$ for any index satisfying properties 1-4 and 5'-8'? In fact, as indicated by the subsequent Theorem 2 , there are. The result is a partial ordering. Like the ordering generated by Lorenz or segregation curves, the $x$ distribution can be declared more segregated than $y$ for some but not all feasible realizations of $x$ and $y$. Additional notation is required to establish these conditions on $x$ and $y$. First, it is necessary to distinguish situations where $k_{i}=k_{j}$ from those where $k_{i}>k_{j}$. Partition the T groups into $\mathrm{G}$ mutually exclusive and exhaustive subsets such that each subset contains one or more groups with the same economic status. Thus, suppose the status of the first $\mathrm{T}_{1}$ groups equals $k_{1}^{*}$, that of the next $\mathrm{T}_{2}$ groups equals $k_{2}^{*}$, and so forth. Then, without loss of generality, rewrite the ordinal ranking as $k_{j g}=k_{g}^{*}, j=1, . ., T_{g}, g=1, . ., G$ where $k_{1}^{*}>k_{2}^{*}>\ldots>k_{G}^{*} \geq 0$

In similar fashion, denote the number of type i people who are in the jth group in subset $g$ as $x_{i j g}$, and let $s_{i j g}^{x}$ indicate the fraction of all type i people in the $x$ distribution who are in the jth group of subset $\mathrm{g}$, thus, $s_{i j g}^{x}=x_{i j g} / N_{i}^{x}, \mathrm{i}=1,2 ; \mathrm{j}=1, . ., T_{g}^{x} ; \mathrm{g}=1, . . \mathrm{G}$. Since the subsequent results utilize the ratio of type 1 to type 2 people, let $q_{j g}^{x}=s_{1 j g}^{x} / s_{2 j g}^{x}$, with $0 \leq q_{j g}^{x} \leq \hat{q}$, where $\hat{q}$ is the maximum value of $q$ in groups with $s_{2 j g}^{x}>0$. 
Now, we wish to establish conditions under which $O(x ; k)-O(y ; k) \geq 0$. Given the results of Theorem 1, write this as $O(x ; k)-O(y ; k)=\Gamma\left\{Z^{x}\right\}-\Gamma\left\{Z^{y}\right\}$, where $\Gamma\{\}$ is a strictly increasing real valued function and $Z^{x}=\sum_{g=1}^{G} \sum_{j=1}^{T_{g}^{x}} L\left(s_{1 j g}^{x}, s_{2 j g}^{x} ; k_{g}^{*}\right)+\lambda\left(k_{g}^{*}\right)\left(s_{1 j g}^{x}+s_{2 j g}^{x}\right)$ with $Z^{y}$ similarly defined. Then since $\Gamma\{\}$ is strictly increasing, the problem is to find conditions under which $\Delta Z \geq 0$ where $\Delta Z=Z^{x}-Z^{y}=\sum_{g=1}^{G} \Delta Z_{g}$ and, $\Delta Z_{g}=\lambda\left(k_{g}^{*}\right)\left[\sum_{j=1}^{T_{g}^{x}}\left(s_{1 j g}^{x}+s_{2 j g}^{x}\right)-\sum_{j=1}^{T_{g}^{y}}\left(s_{1 j g}^{y}+s_{2 j g}^{y}\right)\right]+\sum_{j=1}^{T_{g}^{x}} L\left(s_{1 j g}^{x}, s_{2 j g}^{x} ; k_{g}^{*}\right)-\sum_{j=1}^{T_{g}^{y}} L\left(s_{1 j g}^{y}, s_{2 j g}^{y} ; k_{g}^{*}\right)$.

Introducing additional notation, let $t$ be a non-negative variable and define a function $\rho\left(t, q_{j g}^{x}\right)$ that jumps from zero to one when $t$ becomes greater than or equal to $q_{j g}^{x}$. Thus,

$$
\rho\left(t, q_{j g}^{x}\right)=\left\{\begin{array}{l}
0 \text { if } s_{2 j g}^{x}=0 \\
0 \text { if } s_{2 j g}^{x}>0 \text { and } t<s_{1 j g}^{x} / s_{2 j g}^{x}=q_{j g}^{x} \\
1 \text { if } s_{2 j g}^{x}>0 \text { and } t \geq s_{1 j g}^{x} / s_{2 j g}^{x}=q_{j g}^{x}
\end{array}, \quad \mathrm{j}=1, \ldots, T_{g}^{x} ; \mathrm{g}=1, \ldots, \mathrm{G} .\right.
$$

In addition, let $F_{g}^{x}(t)=\sum_{j=1}^{T_{g}^{x}} s_{2 j g}^{x} \rho\left(t, q_{j g}^{x}\right) / \sum_{j=1}^{T_{g}^{x}} s_{2 j g}^{x}$. Note that $F_{g}^{x}(t)$ behaves like a cumulative function; it indicates the fraction of type 2 people in subset $g$ that are in groups with $q_{j g}^{x} \leq t$. Thus, $F_{g}^{x}(0)=0$, and $F_{g}^{x}(\hat{q})=1$. Finally, define the integral of $F_{g}^{x}(t)$ as, $\varphi_{g}^{x}(q)=\int_{0}^{q} F_{g}^{x}(t) d t=\sum_{j=1}^{T_{g}^{x}} s_{2 j g}^{x} \operatorname{Max}\left[0, q-q_{j g}^{x}\right] / \sum_{j=1}^{T_{g}^{x}} s_{2 j g}^{x}$. In the income inequality literature the integral of the cumulative function, $\varphi()$, is termed the "deficit function."

Similar notation applies to an alternative distribution $y$. Thus, denote the number of type $\mathrm{i}$ people who are in the jth group of subset $\mathrm{g}$ as $y_{i j g}$, and the share of all type i people in the $y$ distribution who are in subset $\mathrm{g}$ as $s_{i j g}^{y}=y_{i j g} / N_{i}^{y}, \mathrm{i}=1,2 ; \mathrm{j}=1, T_{g}^{y} ; \mathrm{g}=1, . . \mathrm{G}$. Let $q_{j g}^{y}=s_{1 j g}^{y} / s_{2 j g}^{y}$, with $0 \leq q_{j g}^{y} \leq \hat{q}$, where $\hat{q}$ is now the maximum value of $q$ in groups with $s_{2 j g}^{\alpha}>0, \alpha=x, y$. Finally, define $F_{j g}^{y}(t)$ and $\varphi_{j g}^{y}(q)$ in a manner parallel to $F_{j g}^{x}(t)$ and $\varphi_{j g}^{x}(q)$. 
The subsequent proof of Theorem 2 shows that using this notation and some algebra, one can rewrite $\Delta Z_{\mathrm{g}}$ as, $\Delta Z_{g}=\alpha_{1 g} C_{g}(\hat{q})+\alpha_{2 g} B_{2 g}-\int_{0}^{\hat{q}}\left[\partial^{2} L\left(q, 1 ; k_{g}^{*}\right) / \partial q^{2}\right] C_{g}(q) d q$, where $\alpha_{1 g}=\lambda\left(k_{g}^{*}\right)+\partial L\left(\hat{q}, 1 ; k_{g}^{*}\right) / \partial q$, $\alpha_{2 g}=\lambda\left(k_{g}^{*}\right)$, and $B_{2 g}=\sum_{j=1}^{T_{g}^{x}} s_{2 j g}^{x}-\sum_{j=1}^{T_{g}^{y}} s_{2 j g}^{y} \cdot{ }^{16}$ With a bit more algebra, suitable restrictions can be placed on the $B_{2 \mathrm{~g}}$ and $C_{\mathrm{g}}(q)$ such that $\Delta \mathrm{Z}=\Sigma_{\mathrm{g}} \Delta \mathrm{Z}_{\mathrm{g}} \geq 0$. Thus, proceeding in a manner analogous to Atkinson and Bourguignon (1987), Bourguignon (1989), and Chambaz and Maurin (1998), the following theorem states conditions on $x$ and $y$ such that $O(x ; k) \geq O(y ; k){ }^{17}$

Theorem 2. Fix the $\mathrm{T}$ groups $1, \ldots, \mathrm{T}$ in descending order of status such that $k_{1}^{*}>k_{2}^{*}>\ldots>k_{G}^{*} \geq 0$ and $k_{j g}=k_{g}^{*}, j=1, \ldots, T_{g} \quad g=1, . ., G$. For all measures of $O($ ) satisfying properties 1-4, 5'-8', the following conditions on $x$ and $y$ are necessary and sufficient for $O(x ; k) \geq O(y ; k)$ :

(9.1) $\sum_{g=1}^{\tau} C_{g}(q) \leq 0$ where $C_{g}(q)=\left(q-\varphi_{g}^{x}(q)\right) \sum_{j=1}^{T_{g}^{x}} s_{2 j g}^{x}-\left(q-\varphi_{g}^{y}(q)\right) \sum_{j=1}^{T_{g}^{y}} s_{2 j g}^{y}$

(9.2) $\sum_{g=1}^{\tau} B_{2 g} \leq 0 \quad$ where $B_{2 g}=\sum_{j=1}^{T_{g}^{x}} s_{2 j g}^{x}-\sum_{j=1}^{T_{g}^{y}} s_{2 j g}^{y}$

for all $q, 0 \leq q \leq \hat{q}$, and $\tau=1, \ldots, G$.

Proof: See Appendix.

While the inequalities in (9.1) and (9.2) are not difficult to verify in actual data (see Section IV below), the necessary condition in the following corollary is useful for purposes of conducting an initial investigation of the data.

Corollary 2.1. For all measures of segregation $O\left(\right.$ ) satisfying properties $1-4,5^{\prime}-8^{\prime}$, and for all measures of economic status $k$ satisfying $k_{1}^{*}>k_{2}^{*}>\ldots>k_{G}^{*} \geq 0$ and $k_{j g}=k_{g}^{*}, j=1, \ldots, T_{g}, g=1, \ldots, G$, the following condition is necessary for $O(x ; k) \geq O(y ; k)$ :

(10) $\sum_{g=1}^{\tau} B_{1 g} \leq 0$ and $\sum_{g=1}^{\tau} B_{2 g} \leq 0, \tau=1, \ldots, G, \quad$ where $B_{i g}=\sum_{j=1}^{T_{g}^{x}} s_{i j g}^{x}-\sum_{j=1}^{T_{g}^{y}} s_{i j g}^{y}, \mathrm{i}=1,2$.

Proof: See Appendix

In other words, begin an analysis of occupational segregation by partitioning the occupations into $\mathrm{G}$ subsets such that all of the occupations within a subset have the same economic status. To 
assess whether $O(x ; k) \geq O(y ; k)$, Corollary 2.1 says that for the $\mathrm{T}_{1}$ groups in the highest status category $(\mathrm{g}=1)$, determine whether the share of type 1 people in the $x$ distribution is smaller than that in the $y$ distribution, i.e., $B_{11}=\sum_{j=1}^{T_{1}^{x}} s_{1 j 1}^{x}-\sum_{j=1}^{T_{1}^{y}} s_{1 j 1}^{y} \leq 0$. Next, for the $\mathrm{T}_{1}+\mathrm{T}_{2}$ groups in the two highest status categories $(\mathrm{g}=1,2)$, determine whether $\sum_{g=1}^{2} B_{1 g}=\sum_{g=1}^{2}\left[\sum_{j=1}^{T_{g}^{x}} s_{1 j g}^{x}-\sum_{j=1}^{T_{g}^{y}} s_{1 j g}^{y}\right] \leq 0$. Compute similar sums for the three highest categories, four highest categories, and so forth, and then do the same for type 2 people. If one of the sums is positive, then the corollary's necessary condition is violated, and it is not the case that $O(x ; k) \geq O(y ; k)$, for all measures of segregation satisfying $1-4,5^{\prime}-8^{\prime}$, and for all measures of economic status $k$ satisfying $k_{1}^{*}>k_{2}^{*}>\ldots>k_{G}^{*} \geq 0$ and $k_{j g}=k_{g}^{*}, j=1, \ldots, T_{g}, g=1, \ldots, G .^{18}$

On the other hand, if the corollary's necessary condition is satisfied, then the analysis shifts to verifying the necessary and sufficient conditions in Theorem 2. This boils down to checking condition (9.1), since verifying Corollary 2.1 requires assessment of the inequalities in (9.2). Condition (9.1) involves a comparison of the deficit functions $(\varphi(q))$ for distributions $x$ and $y$. To see this, consider the case (similar to Atkinson and Bourguignon, 1987) where $\sum_{j=1}^{T_{g}^{x}} s_{2 j g}^{x}=\sum_{j=1}^{T_{g}^{y}} s_{2 j g}^{y}$ for all $\mathrm{g}$. In that case (9.1) says that for the $\mathrm{T}_{1}$ groups in the highest status category $(\mathrm{g}=1)$, determine whether for all $q, C_{1}(q)=\sum_{j=1}^{T_{1}^{x}} s_{2 j 1}^{x}\left[\varphi_{1}^{y}(q)-\varphi_{1}^{x}(q)\right] \leq 0$. Next, for the $\mathrm{T}_{1}+\mathrm{T}_{2}$ groups in the two highest status categories $(\mathrm{g}=1,2)$, determine whether for all $q, \sum_{g=1}^{2} C_{g}(q)=\sum_{g=1}^{2} \sum_{j=1}^{T_{1}^{x}} s_{2 j 1}^{x}\left[\varphi_{1}^{y}(q)-\varphi_{1}^{x}(q)\right] \leq 0$. Compute similar sums for the three highest categories, four highest categories, and so forth. If all of these sums are non-positive for all $q$, then condition (9.1) is satisfied. Matters are somewhat more complicated when $\sum_{j=1}^{T_{g}^{x}} s_{2 j g}^{x} \neq \sum_{j=1}^{T_{g}^{y}} s_{2 j g}^{y}$, but the essential idea is the same. 
In summary, even if there does not exist an agreed upon cardinal measure of economic status, hierarchy can still be incorporated into a measure of segregation. Given an ordinal ranking of occupations, Theorem 2 and its corollary provide a means for declaring distribution $x$ at least as segregated as distribution $y$. The result is a partial ordering; if the conditions of Theorem 2 are not met, then in order to state whether $O(x ; k) \geq O(y ; k)$, the analyst must either accept a cardinal measure of economic status, commit to an explicit measure of segregation, or both. The application in the next section illustrates these points.

This is a good place to address a potential criticism of Theorems 1 and 2: perhaps in combining economic status with variation in numbers of type 1 and 2 people, we end up with a measure of occupational segregation that is less transparent than earlier measures, i.e., a statusaugmented index (such as that in equation (6)) is less transparent than a status-free index (such as (3)). In response, while transparency is desirable, in this situation it comes at a cost; by ignoring economic status, a status-free measure misses a key aspect of what we mean by segregation and is thereby incomplete. The situation is analogous to comparing a headcount measure of poverty with the Foster-Greer-Thorbeck (FGT) measure. ${ }^{19}$ A headcount measure indicates the fraction of a community's population that falls below a poverty line. While this simple measure of poverty is easily understood and enjoys wide use, it is incomplete in that it ignores the extent to which the incomes of the poor fall below the poverty line. In contrast, the FGT measure gets closer to what we mean by poverty in that it includes both the headcount and the gap between incomes and the poverty line. ${ }^{20}$ While the FGT measure may be less transparent than a headcount measure, it is arguably more in accord with what we mean by poverty and thereby more convincing. Similarly, while a status-augmented segregation index may be less transparent than a status-free index, it is arguably more in accord with what we mean by segregation. 
Finally, it is important to note that Theorem 2 is linked to a broader literature; one can show that the results on second order dominance in Atkinson and Bourguignon (1987), Jenkins and Lambert (1993) and Chambaz and Maurin (1998) constitute special cases of Theorem 2. Thus, Theorem 2 is not an isolated result that is cut off from the mainland of inequality research. Rather, it is a more general version of a key theorem in that literature. This point is developed rigorously in a theorem and its attendant (rather lengthy) proof that are available from the author.

\section{An Application}

One way to assess the practical utility of Theorems 1 and 2 is to apply them to data. To what extent do they help us better understand data on segregation? First, consider Theorem 1 and its corollaries. To utilize the equation (6) status-augmented square root index, one must first specify the $2 \mathrm{~T}+3$ parameters, $b, \lambda(\tilde{k}), \gamma(\tilde{k}), \lambda\left(k_{\mathrm{j}}\right)$ and $\gamma\left(k_{\mathrm{j}}\right), \mathrm{j}=1, \ldots, \mathrm{T}$. Matters are simplified by working with the following normalization of the status level $k_{\mathrm{j}}$,

$$
\hat{k}_{j}=\frac{k_{j}-\min \left(k_{j}\right)}{\max \left(k_{j}\right)-\min \left(k_{j}\right)}
$$

where $\min \left(k_{\mathrm{j}}\right)$ and $\max \left(k_{\mathrm{j}}\right)$ are the minimum and maximum values of the $k_{\mathrm{j}}, \mathrm{j}=1, \mathrm{~T}$, and $0 \leq \hat{k}_{j} \leq 1$. In addition, let $\lambda()$ and $\gamma()$ be linear functions of the form, $\gamma\left(k_{j}\right)=\alpha_{\gamma}-\beta_{\gamma}\left[\hat{k}_{j}-\operatorname{med}\left(\hat{k}_{j}\right)\right]$ and $\lambda\left(k_{j}\right)=\alpha_{\lambda}-\beta_{\lambda}\left[\hat{k}_{j}-\operatorname{med}\left(\hat{k}_{j}\right)\right]$ where $\operatorname{med}\left(\hat{k}_{j}\right)$ is the median value of the $\hat{k}_{j}$. As a result, the $2 \mathrm{~T}$ parameters $\lambda\left(k_{\mathrm{j}}\right)$ and $\gamma\left(k_{\mathrm{j}}\right), \mathrm{j}=1, \ldots, \mathrm{T}$ are reduced to four non-negative parameters: $\alpha_{\gamma}, \alpha_{\lambda}, \beta_{\gamma}, \beta_{\lambda}$.

In the subsequent empirical work these four parameters along with $b, \lambda(\tilde{k}), \gamma(\tilde{k})$ are assigned values so as to facilitate interpretation of results. In particular, parameters are specified so that the index takes a minimum value of zero. That occurs when all people are employed in 
occupations with the maximum status and there is complete integration within those occupations $\left(\mathrm{s}_{1 \mathrm{j}}\right.$ $\left.=\mathrm{s}_{2 \mathrm{j}}, \mathrm{j}=1, \ldots, \mathrm{T}\right)$. Moreover, the parameters are specified so that if all people are employed in occupations with the median status $\left(\operatorname{med}\left(\hat{k}_{j}\right)\right)$, then the equation $(6)$ index equals one plus the statusfree square root index. ${ }^{21}$ Call the resulting parameterization of equation (6) "Augmented Index A." This section uses Augmented Index A to analyze occupational segregation by ethnicity and gender in the U.S. Specifically, it measures the degree of occupational segregation from whites for three race/ethnic groups (African American, Hispanic, and Asian), and ranks them from most to least segregated. It also measures occupational segregation of women from men within the four race/ethnic groups (whites, African Americans, Hispanics, and Asians), and asks which group has greater gender segregation.

There exists an extensive empirical literature that addresses such questions. ${ }^{22}$ Particularly noteworthy is a recent analysis of 2000 Census data by David Cotter, Joan Hermsen, and Reeve Vanneman; (2005). Part of their work is mirrored here. Following Cotter, Hermsen, and Vanneman (CHV), the subsequent analysis is restricted to labor force participants age $25-54$ in the U.S. Census 2000 5\% Public Use Micro Data Sample (PUMS). These data provide detailed information on the ethnic and gender composition of 389 occupations. Although it would have been ideal to replicate the CHV results, that proved impossible. ${ }^{23}$

Calculation of a status-augmented measure of segregation requires information on occupational status. While there are several alternatives from which to choose ${ }^{24}$ the following analysis relies on a widely used measure first developed by Charles Nam, Mary Powers, and Monica Boyd. Researchers at the University of Michigan updated the Nam-Powers-Boyd (NPB) index using 1990 data on earnings and education, and then applied it to the Census 2000 PUMS. ${ }^{25}$ Although the 
NPB is scaled from 0 to 100 , for present purposes it is divided into five categories such that the equation (11) normalization takes values $\hat{k}_{1}=1, \hat{k}_{2}=.75 \ldots \hat{k}_{5}=0 .{ }^{26}$

Table 1 introduces the data. Note the differences in economic status. More than $40 \%$ of African American and Hispanic men and women are employed in the bottom two status categories, while more than $40 \%$ of white and Asian men and women are in the top two categories. Tables 2 and 3 present results on occupational segregation. Table 2 examines segregation from whites for African Americans, Hispanics, and Asians. It does this first for males (Panel A), and then for females (Panel B). Table 3 examines gender segregation within the four race/ethnic groups.

Consider Panel A of Table 2. Columns 1 and 3 assess segregation with a pair of status-free measures of segregation: the dissimilarity index and the square root index. As reflected in the rankings in columns 2 and 4, these status-free measures indicate that occupational segregation from white males is more severe for Asian males and less severe for Hispanic and African American males, a result that is consistent with other work on the topic. ${ }^{27}$ Column 5 presents Augmented Index A for these data. We see from the rankings in column 6 that this index paints a different picture of segregation. Now segregation is lowest for Asians (.903) and highest for Hispanics (1.045). Of course, this different picture is in part due to economic status of the jobs held by Asians, Hispanics, and African Americans. In part because African Americans and Hispanics experience their segregation from whites in comparatively low status occupations, the augmented index indicates that their segregation is greater than that for Asians. ${ }^{28}$

Table 3 presents results on segregation of men and women within the four race/ethnic groups (whites, Asians, Hispanics, and African-Americans.) Once again, the augmented square root measure disagrees with the status-free measures. For example, although the column 3 status-free square root 
index declares whites to be more gender segregated than African Americans (.240 versus .202), the column 5 augmented index says that whites are slightly less gender segregated (1.123 versus 1.201).

What about Theorem 2? Do the less restrictive conditions of Theorem 2 yield segregation orderings similar to those above? To keep things simple, the following focuses on the Table 3 gender segregation. The ideas are, of course, equally applicable to the race/ethnic segregation in Table 2.

To implement the conditions in Theorem 2, assume a hierarchy with five levels, and let $k_{1}^{*}$ denote the status of the first (highest) level of the occupational hierarchy, $k_{2}^{*}$ the status for the second highest level of the hierarchy and so forth. For purposes of Theorem 2, the only constraint on the value of the $k_{i}^{*}, \mathrm{i}=1, \ldots, 5$ is that they satisfy the ordinal ranking $k_{1}^{*}>k_{2}^{*}>k_{3}^{*}>k_{4}^{*}>k_{5}^{*} \geq 0$. Of course, the five category scheme used in Tables 1-3 satisfies the constraint. At issue is whether for all measures of status that satisfy the constraint and all measures of segregation that satisfy properties $1-4,5^{\prime}-8$, the four race/ethnic groups can be ranked from most to least gender segregated.

The Corollary 2.1 necessary condition provides a good starting point. For the five levels of the hierarchy in, do we observe $\sum_{g=1}^{\tau} B_{1 g} \leq 0$ and $\sum_{g=1}^{\tau} B_{2 g} \leq 0, \tau=1, \ldots, 5$ ? Consider African Americans and Asians. For the highest level of the hierarchy in Table 1, the percent of African American and Asian women is $17.8 \%$ and $32.3 \%$ respectively. Then $\mathrm{B}_{11}=17.8-32.3=-14.5$. Calculating the other sums for Asian and African American men and women at all five levels of the hierarchy, we find that all sums are non-positive. Thus, the Corollary 2.1 necessary condition is, indeed, satisfied.

The top panel of Table 4 presents gender segregation results for the other race/ethnic groups. The issue here is whether gender segregation for the race/ethnic groups in the columns (the $\mathrm{X}$ race/ethnic groups) is greater than or equal to gender segregation for the race/ethnic groups in the rows (the Y race/ethnic groups). Table 4 indicates that the Corollary 2.1 necessary condition is satisfied in some, but not all, cases. 
When the Corollary 2.1 necessary condition is satisfied, the next step is to evaluate the more elaborate necessary and sufficient condition of Theorem 2 (equations 9.1 and 9.2). As noted in Section III, this reduces to evaluating equation 9.1 since equation 9.2 was already checked when assessing Corollary 2.1. In order to evaluate equation 9.1 a computer program calculated $\sum_{g=1}^{\tau} C_{g}(q)$ for $\tau=1, \ldots, 5$, and for a sequence of 1000 values of $q$ satisfying $0 \leq q \leq \hat{q} \cdot{ }^{29}$ The results are displayed in the lower panel of Table 4. The table indicates that gender segregation among Hispanics exceeds that of both African Americans and Asians, and gender segregation among African Americans exceeds that of Asians. Since the conditions are not satisfied for several other Table 4 pairwise comparisons (e.g., those involving whites), one must impose more stringent assumptions - assumptions that go beyond those in Theorem 2 - to make statements about them.

To conclude, both Theorem 1 and Theorem 2 are useful; they provide a way to look at data on segregation in situations where our notion of segregation includes hierarchical differences in economic status. Moreover, in practice the theorems matter; status-augmented measures of segregation can yield segregation orderings that substantially differ from status-free measures.

\section{Conclusion}

This paper considers the problem of measuring segregation when groups form a hierarchy whereby some groups have greater economic advantage or status than others. The literature has focused on measuring segregation in ways that either ignore economic status or that assume that all groups have the same status. This paper's contribution lies in defining a broader class of segregation measures. The class is not only applicable to the "same economic status" case, but also to cases where people are distributed across groups with different levels of economic status. 
The paper also contributes to the literature by offering two ways to incorporate economic status into empirical work, both of which are utilized in Section IV. One is to rank groups from highest to lowest economic status, and then apply the dominance criteria in Theorem 2 . The other is to decide on a cardinal measure of group economic status and compute a numerical index like the augmented square root index in (6) above. While the first approach has the advantage of requiring less information and weaker value judgments, it comes at the cost of a partial or incomplete ordering. If the conditions in Theorem 2 are met, then we can say that the $x$ distribution is at least as segregated as $y$; if the conditions are not met then, in a manner similar to intersecting Lorenz curves, nothing can be said about segregation of $x$ versus $y$ without additional value judgments.

Application of these ideas in Section IV yields the conclusion that - in contrast to results based on existing status-free measures of segregation - occupational segregation from whites is more severe for African Americans and Hispanics than for Asians. Moreover, gender segregation tends to be greatest among Hispanics versus either African Americans or Asians.

Finally, the paper is part of a broader endeavor whereby the framework used to analyze measures of income inequality is extended to topics other than income (Hutchens 1991, 2001, 2003; Foster, 1994). In particular, the present paper takes ideas from the literature on income inequality among heterogeneous agents, and extends them to the problem of assessing occupational segregation among heterogeneous occupations. Although the literature on segregation has largely developed in isolation from that on income inequality, the two literatures deal with related forms of inequality and can be analyzed with related analytic tools. 
Table 1

Distribution by Economic Status for Men and Women in Four Race/Ethnic Groups

U.S. Census 2000 5\% Public Use Micro Data Sample

\begin{tabular}{|c|c|c|c|c|c|c|c|c|c|c|}
\hline \multirow{3}{*}{$\begin{array}{c}\text { Economic } \\
\text { Status } \\
\text { Category }\end{array}$} & \multirow{3}{*}{$\begin{array}{c}\text { Nam-Powers } \\
\text {-Boyd Index } \\
\text { Average }\end{array}$} & \multirow{3}{*}{$\begin{array}{l}\text { Value } \\
\text { of } k^{\wedge}\end{array}$} & \multicolumn{8}{|c|}{ Race/Ethnic Group } \\
\hline & & & \multicolumn{2}{|c|}{ African American } & \multicolumn{2}{|c|}{ Asian (any) } & \multicolumn{2}{|c|}{ Hispanic (any) } & \multicolumn{2}{|c|}{ White (only) } \\
\hline & & & $\%$ male & \% female & $\%$ male & \% female & $\%$ male & $\%$ female & $\%$ male & $\%$ female \\
\hline$\overline{1 \text { (high) }}$ & 87.09 & 1 & $\overline{15.0 \%}$ & $\overline{17.8 \%}$ & $\overline{40.3 \%}$ & $\overline{32.3 \%}$ & $\overline{11.1 \%}$ & $\overline{13.6 \%}$ & $\overline{27.7 \%}$ & $\overline{26.8 \%}$ \\
\hline 2 & 68.66 & 0.75 & $14.4 \%$ & $15.1 \%$ & $12.8 \%$ & $12.7 \%$ & $10.1 \%$ & $11.9 \%$ & $16.7 \%$ & $17.1 \%$ \\
\hline 3 (middle) & 50.32 & 0.5 & $15.9 \%$ & $20.5 \%$ & $15.2 \%$ & $17.1 \%$ & $15.5 \%$ & $18.5 \%$ & $19.9 \%$ & $22.6 \%$ \\
\hline 4 & 31.29 & 0.25 & $33.4 \%$ & $28.3 \%$ & $18.4 \%$ & $21.6 \%$ & $31.6 \%$ & $25.7 \%$ & $24.3 \%$ & $20.8 \%$ \\
\hline \multirow[t]{2}{*}{5 (low) } & 13.10 & 0 & $21.3 \%$ & $18.3 \%$ & $13.3 \%$ & $16.3 \%$ & $31.7 \%$ & $30.3 \%$ & $11.4 \%$ & $12.7 \%$ \\
\hline & & & $100.0 \%$ & $100.0 \%$ & $100.0 \%$ & $100.0 \%$ & $100.0 \%$ & $100.0 \%$ & $100.0 \%$ & $100.0 \%$ \\
\hline
\end{tabular}

Table 2

Occupational Segregation from Whites for Three Race/Ethnic Groups

Panel A: Men

\begin{tabular}{|c|c|c|c|c|c|c|}
\hline \multirow[b]{2}{*}{$\begin{array}{c}\text { Race/Ethnic } \\
\text { Group }\end{array}$} & \multicolumn{2}{|c|}{$\begin{array}{c}\text { Dissimilarity } \\
\text { Index } \\
\end{array}$} & \multicolumn{2}{|c|}{$\begin{array}{l}\text { Square Root } \\
\text { Index } \\
\end{array}$} & \multicolumn{2}{|c|}{$\begin{array}{l}\text { Augmented } \\
\text { Index A } \\
\end{array}$} \\
\hline & $\begin{array}{c}\text { Value } \\
(1)\end{array}$ & $\begin{array}{c}\text { Rank } \\
\text { (2) }\end{array}$ & $\begin{array}{c}\text { Value } \\
\text { (3) }\end{array}$ & $\begin{array}{c}\text { Rank } \\
(4)\end{array}$ & $\begin{array}{c}\text { Value } \\
(5)\end{array}$ & $\begin{array}{c}\text { Rank } \\
(6)\end{array}$ \\
\hline African American & 0.277 & 1 & 0.057 & 1 & 1.000 & 2 \\
\hline Hispanic & 0.291 & 2 & 0.064 & 2 & 1.045 & 3 \\
\hline Asian & 0.311 & 3 & 0.076 & 3 & 0.903 & 1 \\
\hline
\end{tabular}

Panel B: Women

\begin{tabular}{|c|c|c|c|c|c|c|}
\hline \multirow[b]{2}{*}{$\begin{array}{r}\text { Race/Ethnic } \\
\text { Group }\end{array}$} & \multicolumn{2}{|c|}{$\begin{array}{l}\text { Dissimilarity } \\
\text { Index } \\
\end{array}$} & \multicolumn{2}{|c|}{$\begin{array}{l}\text { Square Root } \\
\text { Index } \\
\end{array}$} & \multicolumn{2}{|c|}{$\begin{array}{l}\text { Augmented } \\
\text { Index A } \\
\end{array}$} \\
\hline & $\begin{array}{c}\text { Value } \\
(1) \\
\end{array}$ & $\begin{array}{c}\text { Rank } \\
(2) \\
\end{array}$ & $\begin{array}{c}\text { Value } \\
\text { (3) }\end{array}$ & $\begin{array}{c}\text { Rank } \\
(4) \\
\end{array}$ & $\begin{array}{c}\text { Value } \\
(5) \\
\end{array}$ & $\begin{array}{c}\text { Rank } \\
(6) \\
\end{array}$ \\
\hline African American & 0.229 & 1 & 0.040 & 1 & 0.993 & 2 \\
\hline Hispanic & 0.249 & 3 & 0.052 & 3 & 1.042 & 3 \\
\hline Asian & 0.243 & 2 & 0.049 & 2 & 0.949 & 1 \\
\hline
\end{tabular}


Table 3

Gender Segregation (women vs. men) within Race/Ethnic Groups

\begin{tabular}{|lccccccc|}
\hline & \multicolumn{2}{c}{$\begin{array}{c}\text { Dissimilarity } \\
\text { Index }\end{array}$} & & \multicolumn{2}{c|}{$\begin{array}{c}\text { Square Root } \\
\text { Index }\end{array}$} & & \multicolumn{2}{c|}{$\begin{array}{c}\text { Augmented } \\
\text { Index A }\end{array}$} \\
\cline { 2 - 6 } Race/Ethnic & Value & Rank & & Value & Rank & Value & Rank \\
Group & $(1)$ & $(2)$ & $(3)$ & $(4)$ & $(5)$ & $(6)$ \\
\hline Asian & 0.415 & 1 & 0.140 & 1 & 0.978 & 1 \\
African American & 0.486 & 2 & 0.202 & 2 & 1.201 & 3 \\
Hispanic & 0.520 & 3 & 0.245 & 4 & 1.310 & 4 \\
White & 0.522 & 4 & 0.240 & 3 & 1.123 & 2 \\
& & & & & & \\
\hline
\end{tabular}

Table 4

Using Theorem 2 and its Corollary to Test for Whether Race/Ethnic Group X is More Gender Segregated than Race/Ethnic Group Y.

Testing the Corollary 2.1 Necessary Condition (NC) for $\mathbf{O}(\mathbf{x} ; \mathbf{k}) \geq \mathbf{O}(\mathbf{y} ; \mathbf{k})$

\begin{tabular}{ccccc|}
\multicolumn{1}{c}{ Race/Ethnic } & \multicolumn{4}{c}{ Race/Ethnic Group X } \\
Group $\mathbf{Y}$ & Hispanic & African-American & White & Asian \\
\cline { 2 - 5 } Hispanic & -- & NC Fails & NC Fails & NC Fails \\
African-American & NC Pass & -- & NC Fails & NC Fails \\
White & NC Pass & NC Pass & -- & NC Fails \\
Asian & NC Pass & NC Pass & NC Fails & -- \\
\cline { 2 - 5 } & &
\end{tabular}

Testing Theorem 2 Necessary and Sufficient Condition $(\mathrm{N \& SC})$ for $\mathrm{O}(\mathrm{x} ; \mathrm{k}) \geq \mathrm{O}(\mathrm{y} ; \mathbf{k})$

\begin{tabular}{|c|c|c|c|c|}
\hline \multirow{2}{*}{$\begin{array}{c}\text { Race/Ethnic } \\
\text { Group Y }\end{array}$} & \multicolumn{4}{|c|}{ Race/Ethnic Group X } \\
\hline & $\underline{\text { Hispanic }}$ & African-American & White & $\underline{\text { Asian }}$ \\
\hline Hispanic & -- & & & \\
\hline African-American & N\&SC Pass & -- & & \\
\hline White & N\&SC Fail & N\&SC Fail & -- & \\
\hline Asian & N\&SC Pass & N\&SC Pass & - & - - \\
\hline
\end{tabular}




\section{Appendix $^{30}$}

It is easiest to begin the proof of Theorem 1 with a more compact notation. Thus, let $J\left(s_{1 j}, s_{2 j} ; k_{j}\right)=L\left(s_{1 j}, s_{2 j} ; k_{j}\right)+\lambda\left(k_{j}\right)\left(s_{1 j}+s_{2 j}\right)$, and restate Theorem 1 as, $O(x ; k)$ satisfies properties $1-4,5,-8$ ' if and only if

$$
O(x ; k)=\Gamma\left\{\sum_{j=1}^{T}\left[J\left(s_{1 j}, s_{2 j} ; k_{j}\right)\right]\right\}
$$

where $s_{i j}=x_{i j} / N_{i}^{x}, \mathrm{i}=1,2 ; \mathrm{j}=1, \ldots, \mathrm{T}, \Gamma\{\}$ is a strictly increasing differentiable function, $\lambda\left(k_{j}\right)$ is a non-increasing differentiable function, and $J()$ is 3 -smooth with
a) $J\left(0,0 ; k_{j}\right)=0, J\left(s_{1 j}, 0 ; k_{j}\right)=\lambda\left(k_{j}\right) s_{1 j}, J\left(0, s_{2 j} ; k_{j}\right)=\lambda\left(k_{j}\right) s_{2 j}, \mathrm{j}=1, \ldots, \mathrm{T}$,
b) in its first two arguments, $J()$ is symmetric, and homogeneous of degree one,
c) $\partial^{2} J() / \partial s_{i j} \partial k_{j} \leq 0, \mathrm{i}=1,2 ; \mathrm{j}=1, \ldots, \mathrm{T}$,
d) $\partial^{3} J() / \partial s_{1 j}^{2} \partial k_{j} \geq 0$ if $s_{2 j}>0$ and $\partial^{3} J() / \partial s_{1 j}^{2} \partial k_{j}=0$ if $s_{2 j}=0, \mathrm{j}=1, \ldots, \mathrm{T}$,
e) $\partial^{2} J() / \partial s_{1 j}^{2} \geq 0$ if $s_{2 j}>0$ and $\partial^{2} J() / \partial s_{1 j}^{2}=0$ if $s_{2 j}=0, \mathrm{j}=1, \ldots, \mathrm{T}$,
f) $\partial^{3} J() / \partial s_{2 j}^{2} \partial k_{j} \geq 0$ if $s_{1 j}>0$ and $\partial^{3} J() / \partial s_{2 j}^{2} \partial k_{j}=0$ if $s_{1 j}=0, \mathrm{j}=1, \ldots, \mathrm{T}$,
g) $\partial^{2} J() / \partial s_{2 j}^{2} \geq 0$ if $s_{1 j}>0$ and $\partial^{2} J() / \partial s_{2 j}^{2}=0$ if $s_{1 j}=0, \mathrm{j}=1, \ldots, \mathrm{T}$.

The following Lemma simplifies the proof of Theorem 1.

\section{Lemma 1.}

a) Given the definition of $O(y ; k)$ in $\mathrm{P} 7$ ' and evaluating derivatives at $d_{\mathrm{y}}=0$,

$$
\partial O(y ; k) / \partial d_{y}=\frac{\Gamma^{\prime}\{\}}{N_{1}} \int_{s_{1 f}}^{s_{1 g}}\left[\partial^{2} J\left(\xi, s_{2 f} ; k_{f}\right) / \partial \xi^{2}\right] d \xi \text {. }
$$

b) Given the definition of $O(y ; k)$ and $O(z ; k)$ in P7' and evaluating derivatives at $d_{\mathrm{y}}=d_{\mathrm{z}}=0$,

$$
\partial O(z ; k) / \partial d_{z}-\partial O(y ; k) / \partial d_{y}=-\frac{\Gamma^{\prime}\{\}}{N_{1}} \int_{k_{h}}^{k_{g}}\left[\partial^{2} J\left(s_{1 g}, s_{2 g} ; k\right) / \partial s_{1 g} \partial k\right] d k
$$

c) Given the definition of $O(y ; k)$ and $O(v ; k)$ in P8' and evaluating derivatives at $d_{\mathrm{y}}=d_{\mathrm{v}}=0$,

$$
\partial O(v ; k) / \partial d_{v}-\partial O(y ; k) / \partial d_{y}=\frac{\Gamma^{\prime}\{\}}{N_{1}} \int_{k_{g}}^{k_{r}} \int_{s_{1 f}}^{s_{1 g}}\left[\partial^{3} J\left(\xi, s_{2 g} ; k\right) / \partial \xi^{2} \partial k\right] d \xi d k
$$

\section{Proof of Lemma 1.}

(a). From P7' we have 


$$
O(y ; k)=\Gamma\left\{\sum_{j \neq f, g} J\left(s_{1 j}, s_{2 j} ; k_{j}\right)+J\left(s_{1 f}-\frac{d_{y}}{N_{1}}, s_{2 f} ; k_{f}\right)+J\left(s_{1 g}+\frac{d_{y}}{N_{1}}, s_{2 g} ; k_{g}\right)\right\} .
$$

Then,

$$
\begin{aligned}
\partial O(y ; k) / \partial d_{y} & =\Gamma^{\prime}\{\}\left[\partial J\left(s_{1 f}-\frac{d_{y}}{N_{1}}, s_{2 f} ; k_{f}\right) / \partial d_{y}+\partial J\left(s_{1 g}+\frac{d_{y}}{N_{1}}, s_{2 g} ; k_{g}\right) / \partial d_{y}\right] \\
& =\frac{\Gamma^{\prime}\{\}}{N_{1}}\left[-\partial J\left(s_{1 f}-\frac{d_{y}}{N_{1}}, s_{2 f} ; k_{f}\right) / \partial s_{1 f}+\partial J\left(s_{1 g}+\frac{d_{y}}{N_{1}}, s_{2 g} ; k_{g}\right) / \partial s_{1 g}\right]
\end{aligned}
$$

Where the last equality is obtained with the chain rule. Then at $d_{y}=0$,

$$
\partial O(y ; k) / \partial d_{y}=\frac{\Gamma^{\prime}\{\}}{N_{1}}\left[-\partial J\left(s_{1 f}, s_{2 f} ; k_{f}\right) / \partial s_{1 f}+\partial J\left(s_{1 g}, s_{2 g} ; k_{g}\right) / \partial s_{1 g}\right] .
$$

Now, $\int_{s_{1 f}}^{s_{1 g}}\left[\partial^{2} J\left(\xi, s_{2 f} ; k_{f}\right) / \partial \xi^{2}\right] d \xi=\partial J\left(s_{1 g}, s_{2 f} ; k_{f}\right) / \partial s_{1 g}-\partial J\left(s_{1 f}, s_{2 f} ; k_{f}\right) / \partial s_{1 f}$.

If, as is assumed in the definition of $O(y ; k)$ in P7', $s_{2 g}=s_{2 f}$ and $k_{g}=k_{f}$, then this can be written, $\int_{s_{1 f}}^{s_{1 g}}\left[\partial^{2} J\left(\xi, s_{2 f} ; k_{f}\right) / \partial \xi^{2}\right] d \xi=\partial J\left(s_{1 g}, s_{2 g} ; k_{g}\right) / \partial s_{1 g}-\partial J\left(s_{1 f}, s_{2 f} ; k_{f}\right) / \partial s_{1 f}$. Substituting into the above expression for $\partial O(y ; k) / \partial d_{y}$, it follows that evaluated at $d_{\mathrm{y}}=0$,

$$
\partial O(y ; k) / \partial d_{y}=\frac{\Gamma^{\prime}\{\}}{N_{1}} \int_{s_{1 f}}^{s_{1 g}}\left[\partial^{2} J\left(\xi, s_{2 f} ; k_{f}\right) / \partial \xi^{2}\right] d \xi .
$$

(b) From P7' we have

$$
O(z ; k)=\Gamma\left\{\sum_{j \neq f, h} J\left(s_{1 j}, s_{2 j} ; k_{j}\right)+J\left(s_{1 f}-\frac{d_{z}}{N_{1}}, s_{2 f} ; k_{f}\right)+J\left(s_{1 h}+\frac{d_{z}}{N_{1}}, s_{2 h} ; k_{h}\right)\right\}
$$

Then,

$$
\begin{aligned}
\partial O(z ; k) / \partial d_{z} & =\Gamma^{\prime}\{\}\left[\partial J\left(s_{1 f}-\frac{d_{z}}{N_{1}}, s_{2 f} ; k_{f}\right) / \partial d_{z}+\partial J\left(s_{1 h}+\frac{d_{z}}{N_{1}}, s_{2 h} ; k_{h}\right) / \partial d_{z}\right] \\
& =\frac{\Gamma^{\prime}\{\}}{N_{1}}\left[-\partial J\left(s_{1 f}-\frac{d_{z}}{N_{1}}, s_{2 f} ; k_{f}\right) / \partial s_{1 f}+\partial J\left(s_{1 h}+\frac{d_{z}}{N_{1}}, s_{2 h} ; k_{h}\right) / \partial s_{1 h}\right]
\end{aligned}
$$

where the last equality is obtained with the chain rule. Evaluating this at $d_{z}=0$,

$$
\partial O(z ; k) / \partial d_{z}=\frac{\Gamma^{\prime}\{\}}{N_{1}}\left[-\partial J\left(s_{1 f}, s_{2 f} ; k_{f}\right) / \partial s_{1 f}+\partial J\left(s_{1 h}, s_{2 h} ; k_{h}\right) / \partial s_{1 h}\right] \cdot
$$

From Lemma 1(a), $\partial O(y ; k) / \partial d_{y}$ evaluated at $d_{y}=0$ yields,

$$
\partial O(y ; k) / \partial d_{y}=\frac{\Gamma^{\prime}\{\}}{N_{1}}\left[-\partial J\left(s_{1 f}, s_{2 f} ; k_{f}\right) / \partial s_{1 f}+\partial J\left(s_{1 g}, s_{2 g} ; k_{g}\right) / \partial s_{1 g}\right]
$$

Then at $d_{y}=d_{z}=0$ 


$$
\partial O(z ; k) / \partial d_{z}-\partial O(y ; k) \partial d_{y}=\frac{\Gamma^{\prime}\{\}}{N_{1}}\left[\partial J\left(s_{1 h}, s_{2 h} ; k_{h}\right) / \partial s_{1 h}-\partial J\left(s_{1 g}, s_{2 g} ; k_{g}\right) / \partial s_{1 g}\right] .
$$

Now $\int_{k_{h}}^{k_{g}}\left[\partial^{2} J\left(s_{1 g}, s_{2 g} ; k\right) / \partial s_{1 g} \partial k\right] d k=\partial J\left(s_{1 g}, s_{2 g} ; k_{g}\right) / \partial s_{1 g}-\partial J\left(s_{1 g}, s_{2 g} ; k_{h}\right) / \partial s_{1 g}$. If, as is assumed in the definition of $O(y ; k)$ and $O(z ; k)$ in P7', $s_{1 g}=s_{1 h}$ and $s_{2 g}=s_{2 h}$, then this can be written:

$$
\int_{k_{h}}^{k_{g}}\left[\partial^{2} J\left(s_{1 g}, s_{2 g} ; k\right) / \partial s_{1 g} \partial k\right] d k=\partial J\left(s_{1 g}, s_{2 g} ; k_{g}\right) / \partial s_{1 g}-\partial J\left(s_{1 h}, s_{2 h} ; k_{h}\right) / \partial s_{1 h} .
$$

It follows that, evaluated at $d_{y}=d_{z}=0$

$$
\begin{aligned}
\partial O(z ; k) / \partial d_{z}-\partial O(y ; k) \partial d_{y} & =\frac{\Gamma^{\prime}\{\}}{N_{1}}\left[\partial J\left(s_{1 h}, s_{2 h} ; k_{h}\right) / \partial s_{1 h}-\partial J\left(s_{1 g}, s_{2 g} ; k_{g}\right) / \partial s_{1 g}\right] \\
& =-\frac{\Gamma^{\prime}\{\}}{N_{1}} \int_{k_{h}}^{k_{g}}\left[\partial^{2} J\left(s_{1 g}, s_{2 g} ; k\right) / \partial s_{1 g} \partial k\right] d k .
\end{aligned}
$$

(c) From P8' we have

$$
O(v ; k)=\Gamma\left\{\sum_{j \neq q, r} J\left(s_{1 j}, s_{2 j} ; k_{j}\right)+J\left(s_{1 q}-\frac{d_{v}}{N_{1}}, s_{2 q} ; k_{q}\right)+J\left(s_{1 r}+\frac{d_{v}}{N_{1}}, s_{2 r} ; k_{r}\right)\right\}
$$

Then arguing in a manner identical to (a) and (b) above, at $d_{v}=0$

$$
\partial O(v ; k) / \partial d_{v}=\frac{\Gamma^{\prime}\{\}}{N_{1}}\left[-\partial J\left(s_{1 q}, s_{2 q} ; k_{q}\right) / \partial s_{1 q}+\partial J\left(s_{1 r}, s_{2 r} ; k_{r}\right) / \partial s_{1 r}\right]
$$

From Lemma 1 (a) $\partial O(y ; k) / \partial d_{y}$ evaluated at $d_{y}=0$ is,

$$
\partial O(y ; k) / \partial d_{y}=\frac{\Gamma^{\prime}\{\}}{N_{1}}\left[-\partial J\left(s_{1 f}, s_{2 f} ; k_{f}\right) / \partial s_{1 f}+\partial J\left(s_{1 g}, s_{2 g} ; k_{g}\right) / \partial s_{1 g}\right]
$$

If, as is assumed in the definition of $O(v ; k)$ and $O(y ; k)$ in $\mathrm{P} 8$ '

$$
\begin{gathered}
s_{1 f}=s_{1 q}, s_{1 g}=s_{1 r}, s_{2 f}=s_{2 g}=s_{2 q}=s_{2 r}, k_{f}=k_{g} \text { and } k_{q}=k_{r} \text { and evaluating at } d_{v}=d_{y}=0 \\
\partial O(v ; k) / \partial d_{v}-\partial O(y ; k) / \partial d_{y}=\frac{\Gamma^{\prime}\{\}}{N_{1}}\left[\partial J\left(s_{1 g}, s_{2 g} ; k_{r}\right) / \partial s_{1 g}-\partial J\left(s_{1 f}, s_{2 f} ; k_{r}\right) / \partial s_{1 f}\right. \\
\left.-\partial J\left(s_{1 g}, s_{2 g} ; k_{g}\right) / \partial s_{1 g}+\partial J\left(s_{1 f}, s_{2 f} ; k_{g}\right) / \partial s_{1 f}\right]
\end{gathered}
$$

Since it is assumed in the definition of $O(v ; k)$ and $O(y ; k)$ that $k_{g}<k_{r}$ and $s_{1 f}<s_{1 g}$, it follows that,

$$
\begin{aligned}
\int_{k_{g}}^{k_{r}} \int_{s_{1 f}}^{s_{1 g}}\left[\partial^{3} J\left(\xi, s_{2 g} ; k\right) / \partial \xi^{2} \partial k\right] d \xi d k & \\
& =\partial J\left(s_{1 g}, s_{2 g} ; k_{r}\right) / \partial s_{1 g}-\partial J\left(s_{1 f}, s_{2 g} ; k_{r}\right) / \partial s_{1 f}-\left[\partial J\left(s_{1 g}, s_{2 g} ; k_{g}\right) / \partial s_{1 g}-\partial J\left(s_{1 f}, s_{2 g} ; k_{g}\right) / \partial s_{1 f}\right] \\
& =\partial J\left(s_{1 g}, s_{2 g} ; k_{r}\right) / \partial s_{1 g}-\partial J\left(s_{1 f}, s_{2 f} ; k_{r}\right) / \partial s_{1 f}-\partial J\left(s_{1 g}, s_{2 g} ; k_{g}\right) / \partial s_{1 g}+\partial J\left(s_{1 f}, s_{2 f} ; k_{g}\right) / \partial s_{1 f}
\end{aligned}
$$

where the last equality is due to $s_{2 f}=s_{2 g}$. Then evaluated at $d_{v}=d_{y}=0$, 


$$
\begin{aligned}
\partial O(v ; k) / \partial d_{v}-\partial O(y ; k) / \partial d_{y}= & \frac{\Gamma^{\prime}\{\}}{N_{1}}\left[\partial J\left(s_{1 g}, s_{2 g} ; k_{r}\right) / \partial s_{1 g}-\partial J\left(s_{1 f}, s_{2 f} ; k_{r}\right) / \partial s_{1 f}\right. \\
& \left.-\partial J\left(s_{1 g}, s_{2 g} ; k_{g}\right) / \partial s_{1 g}+\partial J\left(s_{1 f}, s_{2 f} ; k_{g}\right) / \partial s_{1 f}\right] \\
= & \frac{\Gamma^{\prime}\{\}}{N_{1}} \int_{k_{g}}^{k_{k}} \int_{s_{1 f}}^{s_{1 g}}\left[\partial^{3} J\left(\xi, s_{2 g} ; k\right) / \partial \xi^{2} \partial k\right] d \xi d k .
\end{aligned}
$$

Proof of Theorem 1.

Sufficiency: If $O(x ; k)$ satisfies properties $1-4,5^{\prime}-8$, then it takes the form of (A.1).

Let $\bar{O}(x ; k)=\Gamma^{-1}\{O(x ; k)\}$. In accordance with P5, $\bar{O}(x ; k)$ takes the form of (A.2)

$$
\bar{O}(x ; k)=\sum_{j=1}^{T} O^{j}\left(x_{1 j}, x_{2 j} ; k_{j}\right) .
$$

Then the proof of sufficiency proceeds in several steps.

Step 1. P4 and P6' imply that $O^{j}\left(x_{1 j}, x_{2 j} ; k^{0}\right)$ is symmetric in its first two arguments for $\mathrm{j}=$ $1, \ldots, \mathrm{T}$, where $k^{0}=\left[k_{1}^{0}, k_{2}^{0}, \ldots, k_{T}^{0}\right]$ and $k_{1}^{0}=k_{2}^{0}=\ldots=k_{T}^{0}$.

Let $x_{1 T} \neq x_{2 T}$ and $x_{1 j}=x_{2 j}, \mathrm{j}=1, \mathrm{~T}-1$. Then P4 implies, $\bar{O}\left(x ; k^{0}\right)=\sum_{j=1}^{T} O^{j}\left(x_{1 j}, x_{2 j} ; k_{j}^{0}\right)=\bar{O}\left(y ; k^{0}\right)=\sum_{j=1}^{T-1} O^{j}\left(x_{2 j}, x_{1 j} ; k_{j}^{0}\right)+O^{T}\left(x_{2 T}, x_{1 T} ; k_{T}^{0}\right)$.

Since $x_{1 j}=x_{2 j}, \mathrm{j}=1, \ldots, \mathrm{T}-1$, it follows that $O^{T}\left(x_{1 T}, x_{2 T} ; k_{T}^{0}\right)=O^{T}\left(x_{2 T}, x_{1 T} ; k_{T}^{0}\right)$. Then $O^{T}$ is symmetric, and by $\mathrm{P} 6$ '(symmetry in groups), $O^{j}\left(x_{1 j}, x_{2 j} ; k_{j}^{0}\right)$ is symmetric for $\mathrm{j}=1, \ldots \mathrm{T}$.

Step 2. $\mathrm{P} 3$ and $\mathrm{P} 6$ ' imply that if $x_{1 j}=x_{2 j}=0$ then $O^{j}\left(x_{1 j}, x_{2 j} ; k_{j}^{0}\right)=0$.

Let $x_{1 T}=x_{2 T}=0$. Then $\mathrm{P} 3$ implies $\bar{O}\left(x ; k^{0}\right)=\sum_{j=1}^{T} O^{j}\left(x_{1 j}, x_{2 j} ; k_{j}^{0}\right)=\bar{O}\left(y ; k^{0}\right)=\sum_{j=1}^{T-1} O^{j}\left(x_{1 j}, x_{2 j} ; k_{j}^{0}\right)$. So $O^{T}\left(x_{2 T}, x_{1 T} ; k_{T}^{0}\right)=0$. Then by P6' (symmetry in groups) $x_{1 j}=x_{2 j}=0$ implies $O^{j}\left(x_{1 j}, x_{2 j} ; k_{j}^{0}\right)=0$.

Step 3. P3 and P6' imply that $O^{\mathrm{g}}\left(x_{1 \mathrm{~g}}, x_{2 \mathrm{~g}} ; k_{g}^{0}\right)=O^{\mathrm{h}}\left(x_{1 \mathrm{~g}}, x_{2 g} ; k_{g}^{0}\right)$ for all $\mathrm{g}, \mathrm{h}=1, \ldots, \mathrm{T}$.

Assume that distribution $y$ is identical to $x$ except that the people in groups $\mathrm{g}$ and $\mathrm{h}$ trade places. Then $y_{1 g}=x_{1 h}, y_{2 g}=x_{2 h}, y_{1 h}=x_{1 g}, y_{2 h}=x_{2 g}$, and $y_{1 j}=x_{\mathrm{ij}}, \mathrm{i}=1,2$ and $\mathrm{j}=1, \ldots, \mathrm{T}, \mathrm{j} \neq \mathrm{g}, \mathrm{h}$. By P6', $O\left(x ; k^{0}\right)=O\left(y ; k^{0}\right)$. Then given A.2, P6' implies,

(A.3) $O^{\mathrm{g}}\left(x_{1 g}, x_{2 g} ; k_{g}^{0}\right)+O^{\mathrm{h}}\left(x_{1 h}, x_{2 h} ; k_{g}^{0}\right)=O^{\mathrm{g}}\left(y_{1 g}, y_{2 g} ; k_{g}^{0}\right)+O^{\mathrm{h}}\left(y_{1 h}, y_{2 h} ; k_{g}^{0}\right)$.

Since that is true for all feasible values of $x_{i j}$, consider $x_{1 h}=x_{2 h}=y_{1 g}=y_{2 g}=0$. From step 2 and P3 we get that $O^{\mathrm{h}}\left(x_{1 h}, x_{2 h} ; k_{g}^{0}\right)=O^{\mathrm{g}}\left(y_{1 g}, y_{2 g} ; k_{g}^{0}\right)=0$. Then from A.3 it follows that 
$O^{\mathrm{g}}\left(x_{1 g}, x_{2 g} ; k_{g}^{0}\right)=O^{\mathrm{h}}\left(y_{1 h}, y_{2 h} ; k_{g}^{0}\right)$. Therefore, since $y_{2 h}=x_{2 g}$ and $y_{1 h}=x_{1 g}$ it is the case that $O^{\mathrm{g}}\left(x_{1 g}, x_{2 g} ; k_{g}^{0}\right)=O^{\mathrm{h}}\left(x_{1 g}, x_{2 g} ; k_{g}^{0}\right), \mathrm{g}, \mathrm{h}=1, \ldots, \mathrm{T}$.

Step 4. P1, P2, P3, P5' and P6' imply that

$$
\bar{O}(x ; k)=\sum_{j=1}^{T} J\left(s_{1 j}, s_{2 j} ; k_{j}\right)
$$

where $s_{1 j}=x_{1 j} / \mathrm{N}_{1}, s_{2 j}=x_{2 j} / \mathrm{N}_{2}, J\left(s_{1 j} ; s_{2 j} ; k_{j}\right)$ is 3-smooth and, in its first two arguments, symmetric and homogeneous of degree one with $J\left(0,0 ; k_{j}\right)=0, J\left(s_{1 j}, 0 ; k_{j}\right)=\lambda\left(k_{j}\right) \mathrm{s}_{1 j}$, and $J\left(0, s_{2 j} ; k_{j}\right)=\lambda\left(k_{j}\right) s_{2 j}$.

$$
\begin{aligned}
& \text { From P1, } \bar{O}(x ; k)=\sum_{j=1}^{T} O^{j}\left(x_{1 j}, x_{2 j} ; k^{0}\right)=\sum_{j=1}^{T} O^{j}\left(\alpha x_{1 j}, \beta x_{2 j} ; k^{0}\right), \alpha>0, \beta>0 \text {. Letting } \\
& \alpha=1 / N_{1}=1 / \sum_{j=1}^{T} x_{1 j} \text { and } \beta=1 / N_{2}=1 / \sum_{j=1}^{T} x_{2 j}, \text { it follows that } \\
& \bar{O}(x ; k)=\sum_{j=1}^{T} O^{j}\left(x_{1 j}, x_{2 j} ; k^{0}\right)=\sum_{j=1}^{T} O^{j}\left(s_{1 j}, s_{2 j} ; k^{0}\right)=\sum_{j=1}^{T} J\left(s_{1 j}, s_{2 j} ; k^{0}\right)
\end{aligned}
$$

where (using the result in Step 3), $J\left(s_{1 j}, s_{2 j} ; k^{0}\right)=O^{j}\left(s_{1 j}, s_{2 j} ; k^{0}\right)=O^{h}\left(s_{1 j}, s_{2 j} ; k^{0}\right)$, $j=1, \ldots, \mathrm{T} ; h=1, \ldots, \mathrm{T}$. If $O(x ; k)$ satisfies $\mathrm{P} 2$, then,

$$
\bar{O}\left(y ; k^{0}\right)=\sum_{j=1}^{T-1} J\left(s_{1 j}, s_{2 j} ; k^{0}\right)+M J\left(s_{1 T} / M, s_{2 T} / M ; k^{0}\right)=\sum_{j=1}^{T} J\left(s_{1 j}, s_{2 j} ; k^{0}\right)=\bar{O}\left(x ; k^{0}\right),
$$

implying $M J\left(s_{1 T} / M, s_{2 T} / M ; k^{0}\right)=J\left(s_{1 T}, s_{2 T} ; k^{0}\right)$. Let $K$ be a positive integer, then

$$
\begin{aligned}
& M J\left(s_{1 T} K / M, s_{2 T} K / M ; k^{0}\right)=J\left(s_{1 T} K, s_{2 T} K ; k^{0}\right)=K J\left(s_{1 T}, s_{2 T} ; k^{0}\right), \text { and } \\
& \frac{M}{K} J\left(s_{1 T} K / M, s_{2 T} K / M ; k^{0}\right)=J\left(s_{1 T}, s_{2 T} ; k^{0}\right) .
\end{aligned}
$$

For any nonnegative real number $\sigma$ there is a sequence of rational positive numbers $\sigma_{j} \rightarrow \sigma$. Applying the previous result to $\sigma_{j}$, taking the limit $\sigma_{j} \rightarrow \sigma$ and using continuity of $J$, we conclude that $J$ is homogeneous of degree one in its first two arguments. From step $1 J$ is symmetric in its first two arguments, and from step $2 J\left(0,0 ; k_{j}^{0}\right)=0$. Given that $J()$ is symmetric in its first two arguments, let $J\left(1,0 ; k_{j}^{0}\right)=J\left(0,1 ; k_{j}^{0}\right)=\lambda\left(k_{j}^{0}\right)$. Since $J()$ is homogeneous of degree one in its first two arguments, $J\left(s_{1 j}, 0 ; k_{j}^{0}\right)=s_{1 j} J\left(1,0 ; k_{j}^{0}\right)=\lambda\left(k_{j}^{0}\right) s_{1 j}$ and $J\left(0, s_{2 j} ; k_{j}^{0}\right)=s_{2 j} J\left(0,1 ; k_{j}^{0}\right)=\lambda\left(k_{j}^{0}\right.$ )$s_{2 j}$. Then A.4 is valid for $k^{0}$.

Designate a new $k$ vector as $k^{\prime}$ where $k_{T}^{\prime}=k_{T}^{0}$ and the $k_{j}^{\prime} \leq k_{j}^{0}, \mathrm{j}=1, \ldots, \mathrm{T}-1$. Since $k_{T}^{\prime}=k_{T}^{0}$, it follows that $J\left(s_{1 T}, s_{2 T} ; k_{T}^{\prime}\right)=J\left(s_{1 T}, s_{2 T} ; k_{T}^{0}\right)$ for all feasible values of $k_{j}^{\prime}, \mathrm{j}=1, \mathrm{~T}-1$. Then $J\left(s_{1 T}, s_{2 T} ; k_{T}^{\prime}\right)$ is symmetric and homogeneous of degree one in its first two arguments, with $J\left(0,0 ; k_{T}^{\prime}\right)=0, J\left(s_{1 T}, 0 ; k_{T}^{\prime}\right)=\lambda\left(k_{T}^{\prime}\right) \mathrm{s}_{1 T}$, and $J\left(0, s_{2 T} ; k_{T}^{\prime}\right)=\lambda\left(k_{T}^{\prime}\right) s_{2 T}, \mathrm{j}=1, \ldots, \mathrm{T}$. Since the index in A.1 is additive in groups (P5') and symmetric in groups (P6'), it follows that for all feasible 
values of the vector $k, J\left(s_{1 j}, s_{2 j} ; k_{j}\right)$ is symmetric and homogeneous of degree one in its first two arguments, with $J\left(0,0 ; k_{j}\right)=0, J\left(s_{1 j}, 0 ; k_{j}\right)=\lambda\left(k_{j}\right) s_{1 j}$, and $J\left(0, s_{2 j} ; k_{j}\right)=\lambda\left(k_{j}\right) s_{2 j}, \mathrm{j}=1, \ldots, \mathrm{T}$. Moreover, from property $\mathrm{P} 5, J()$ is 3-smooth.

Step 5. P1 - P4, P5' - P7' imply part (c) of Theorem 1, i.e.,

$$
\partial^{2} J\left(s_{1 j}, s_{2 j} ; k_{j}\right) / \partial s_{1 j} \partial k_{j} \leq 0 \text { and } \partial^{2} J\left(s_{1 j}, s_{2 j} ; k_{j}\right) / \partial s_{2 j} \partial k_{j} \leq 0 \mathrm{j}=1, \ldots, \mathrm{T} \text {. }
$$

From P7'(a) and (b), if $x_{2 f}=x_{2 g}=x_{2 h} \geq 0$ then $O(x ; k) \leq O(y ; k) \leq O(z ; k)$ for all feasible $d_{\mathrm{y}}$ $=d_{\mathrm{z}}$. Then taking derivatives with respect to $d_{\mathrm{y}}$ and $d_{\mathrm{z}}$, and evaluating at $d_{\mathrm{y}}=d_{\mathrm{z}}=0$,

$0 \leq \partial O(y ; k) / \partial d_{y} \leq \partial O(z ; k) / \partial d_{z}$. It follows that $\partial O(z ; k) / \partial d_{z}-\partial O(y ; k) / \partial d_{y} \geq 0$, which from Lemma $1 \mathrm{~b}$ implies,

$$
-\frac{\Gamma^{\prime}\{\}^{\prime}}{N_{1}} \int_{k_{h}}^{k_{g}}\left[\partial^{2} J\left(s_{1 g}, s_{2 g} ; k\right) / \partial s_{1 j} \partial k\right] d k \geq 0 .
$$

Now, we wish to establish that $\partial^{2} J\left(s_{1 j}, s_{2 j} ; k_{j}\right) / \partial s_{1 j} \partial k_{j} \leq 0$. Suppose to the contrary that $\partial^{2} J\left(s_{1 j}, s_{2 j} ; k_{j}\right) / \partial s_{1 j} \partial k_{j}>0$ for some $s_{1 j}, s_{2 j}$, and $k_{j}$. Then since $J()$ is a 3 -smooth function, it must be that $\frac{\Gamma^{\prime}\{\}^{k_{j}+\varepsilon}}{N_{1}} \int_{k_{j}-\varepsilon}^{k^{-\varepsilon}}\left[\partial^{2} J\left(s_{1 j}, s_{2 j} ; k\right) / \partial s_{1 j} \partial k\right] d k>0$ for some $\varepsilon>0$. But that contradicts A.5. Then $\partial^{2} J\left(s_{1 j}, s_{2 j} ; k_{j}\right) / \partial s_{1 j} \partial k_{j} \leq 0, \mathrm{j}=1, \ldots, \mathrm{T}$.

To show that $\partial^{2} J\left(s_{1 j}, s_{2 j} ; k_{j}\right) / \partial s_{2 j} \partial k_{j} \leq 0$, note that from Step $4 J()$ is symmetric, and thus, $J\left(a, b ; k_{j}\right)=J\left(b, a ; k_{j}\right)$ for all $a \geq 0$ and $b \geq 0$. It follows that $\partial^{2} J\left(a, b ; k_{j}\right) / \partial a \partial k_{j}=$ $\partial^{2} J\left(b, a ; k_{j}\right) / \partial a \partial k_{j}$ for all $a \geq 0$ and $b \geq 0$. Then $\partial^{2} J\left(s_{1 j}, s_{2 j} ; k_{j}\right) / \partial s_{1 j} \partial k_{j} \leq 0$ implies $\partial^{2} J\left(s_{1 j}, s_{2 j} ; k_{j}\right) / \partial s_{2 j} \partial k_{j} \leq 0, \mathrm{j}=1, \ldots, \mathrm{T}$.

Step 6. P1 - P4, P5' - P7' imply part (e) and (g) of Theorem 1, i.e.,

$$
\begin{aligned}
& \partial^{2} J() / \partial s_{1 j}^{2} \geq 0 \text { if } s_{2 j}>0 \text { and } \partial^{2} J() / \partial s_{1 j}^{2}=0 \text { if } s_{2 j}=0, \mathrm{j}=1, \ldots, \mathrm{T} . \\
& \partial^{2} J() / \partial s_{2 j}^{2} \geq 0 \text { if } s_{1 j}>0 \text { and } \partial^{2} J() / \partial s_{2 j}^{2}=0 \text { if } s_{1 j}=0, \mathrm{j}=1, \ldots, \mathrm{T} .
\end{aligned}
$$

From P7'(a), if $x_{2 f}=x_{2 g}=x_{2 h}>0$ then $O(x ; k) \leq O(y ; k)$ for all feasible $d_{\mathrm{y}}$. Then taking derivatives with respect to $d_{\mathrm{y}}$ and evaluating at $d_{\mathrm{y}}=0,0 \leq \partial O(y ; k) / \partial d_{y}$. It follows from Lemma 1a that for $s_{2 f}>0$

(A.6) $\frac{\Gamma^{\prime}\{\}}{N_{1}} \int_{s_{1 f}}^{s_{1 g}}\left[\partial^{2} J\left(\xi, s_{2 f} ; k_{f}\right) / \partial \xi^{2}\right] d \xi \geq 0$.

Now, we wish to establish that $\partial^{2} J() / \partial s_{1 j}^{2} \geq 0$. Suppose to the contrary that $\partial^{2} J() / \partial s_{1 j}^{2}<0$, for some $s_{1 j}, k_{j}$ and $s_{2 j}>0$. Then since $J()$ is a 3 -smooth function it must be that 
$\frac{\Gamma^{\prime}\{\}^{\prime}}{N_{1}} \int_{s_{1 j}-\delta}^{s_{1 j}+\delta}\left[\partial^{2} J\left(\xi, s_{2 j} ; k_{j}\right) / \partial \xi^{2}\right] d \xi<0$ for some $\delta>0$. But that contradicts (A.6). Then $\partial^{2} J() / \partial s_{1 j}^{2} \geq 0$ if $s_{2 j}>0, j=1, \ldots, T$.

From P7'(b), if $x_{2 f}=x_{2 g}=x_{2 h}=0$ then $0=\partial O(y ; k) / \partial d_{y}$. Proceeding in the same manner as the above treatment of P7'(a), it is straightforward to show that, $\partial^{2} J() / \partial s_{1 j}^{2}=0$ if $s_{2 j}=0, \mathrm{j}=$ $1, \ldots, \mathrm{T}$.

As in Step 5, symmetry of $J()$ implies $\partial^{2} J() / \partial s_{2 j}^{2} \geq 0$ if $s_{1 j}>0$, and $\partial^{2} J() / \partial s_{2 j}^{2}=0$ if $s_{1 j}=$ $0, j=1, \ldots, T$.

Step 7. P1 - P4, P5' - P8' imply part (d) and (f) of Theorem 1, i.e.,

$$
\begin{aligned}
& \partial^{3} J() / \partial s_{1 j}^{2} \partial k_{j} \geq 0 \text { if } s_{2 j}>0 \text { and } \partial^{3} J() / \partial s_{1 j}^{2} \partial k_{j}=0 \text { if } s_{2 j}=0, \mathrm{j}=1, \ldots, \mathrm{T} . \\
& \partial^{3} J() / \partial s_{2 j}^{2} \partial k_{j} \geq 0 \text { if } s_{1 j}>0 \text { and } \partial^{3} J() / \partial s_{2 j}^{2} \partial k_{j}=0 \text { if } s_{1 j}=0, \mathrm{j}=1, \ldots, \mathrm{T} .
\end{aligned}
$$

From P8'(a), if $x_{2 f}=x_{2 g}=x_{2 q}=x_{2 r}>0$ then $\mathrm{O}(x ; k) \leq \mathrm{O}(y ; k) \leq \mathrm{O}(v ; k)$ for all feasible $d_{\mathrm{y}}$ $=d_{\mathrm{v}}$. Then taking derivatives with respect to $d_{\mathrm{v}}$ and $d_{\mathrm{y}}$, and evaluating at $d_{\mathrm{y}}=d_{\mathrm{v}}=0$, $0 \leq \partial O(y ; k) / \partial d_{y} \leq \partial O(v ; k) / \partial d_{v}$, which implies $\partial O(v ; k) / \partial d_{v}-\partial O(y ; k) / \partial d_{y} \geq 0$. It follows from Lemma 1c that for $s_{2 g}>0$,

$$
\frac{\Gamma^{\prime}\{\}}{N_{1}} \int_{k_{g}}^{k_{r}} \int_{s_{1 f}}^{s_{1 g}}\left[\partial^{3} J\left(\xi, s_{2 g} ; k\right) / \partial \xi^{2} \partial k\right] d \xi d k \geq 0 .
$$

Now, we wish to establish that $\partial^{3} J() / \partial s_{1 j}^{2} \partial k_{j} \geq 0$. Suppose to the contrary that $\partial^{3} J() / \partial s_{1 j}^{2} \partial k_{j}<0$, for some $s_{1 j}, k_{j}$, and $s_{2 j}>0$. Then since $J()$ is a 3 -smooth function it must be that $\frac{\Gamma^{\prime}\{\}^{\prime}}{N_{1}} \int_{k_{j}-\varepsilon s_{1_{j}}-\delta}^{k_{j}+\varepsilon s_{1_{j}}+\delta}\left[\partial^{3} J\left(\xi, s_{2 j} ; k\right) / \partial \xi^{2} \partial k\right] d \xi d k<0$ for some $\varepsilon>0$ and $\delta>0$. But that contradicts (A.7). Then $\partial^{3} J() / \partial s_{1 j}^{2} \partial k_{j} \geq 0$ if $s_{2 j}>0, j=1, \ldots, T$.

From Property $8^{\prime}(\mathrm{b})$, if $x_{2 f}=x_{2 g}=x_{2 q}=x_{2 r}=0$ then $\mathrm{O}(x ; k)=\mathrm{O}(y ; k)=\mathrm{O}(v ; k)$. Proceeding in the same manner as the above proof from Property $8^{\prime}(a)$, it is straightforward to show that, $\partial^{3} J() / \partial s_{1 j}^{2} \partial k_{j}=0$ if $s_{2 j}=0, \mathrm{j}=1, \ldots, \mathrm{T}$.

As in Step 5, symmetry of $J()$ implies $\partial^{3} J() / \partial s_{2 j}^{2} \partial k_{j} \geq 0$ if $s_{1 j}>0$ and $\partial^{3} J() / \partial s_{2 j}^{2} \partial k_{j}=0$ if $s_{1 \mathrm{j}}=0, \mathrm{j}=1, \ldots, \mathrm{T}$.

Step 8. $\lambda\left(k_{j}\right)$ is non-increasing in $k_{j}$.

From Theorem 1(a), $J\left(s_{1 j}, 0 ; k_{j}\right)=\lambda\left(k_{j}\right) s_{1 j}$. From Theorem $1(\mathrm{c}), \partial^{2} J() / \partial s_{1 j} \partial k_{j} \leq 0$. Then $\lambda\left(k_{j}\right)$ is nonincreasing in $k_{j}$.

Necessity: If $O(x ; k)$ is of the form of (A.1), where $\Gamma\{\}$ is a continuous and strictly increasing function, and $J()$ is a 3 -smooth function with properties (a) - (g) in this appendix's restatement of Theorem 1, then $O(x ; k)$ satisfies properties 1 - 4, 5' - 8'. 
Proof: P1 (scale invariance) follows from the fact that $x_{\mathrm{i}} / \mathrm{N}_{\mathrm{i}}$ is invariant to a proportional change in $x_{\mathrm{i} 1}, \ldots, x_{\mathrm{i} T}$. P2 (insensitivity to proportional divisions) is proved by obtaining $y$ from $x$ by a proportional division of the Tth group into M subgroups and using $\mathrm{J}($ )'s homogeneity of degree one. P3 (zero member independence) is proved by taking $y$ identical to $x$ except that $y$ excludes group T where $x_{1 T}=x_{2 T}=0$, and using $J\left(0,0 ; k_{T}\right)=0$. To show P4 (symmetry in types) let $x=\left[\begin{array}{l}x_{1} \\ x_{2}\end{array}\right]$ and $y=\left[\begin{array}{l}x_{2} \\ x_{1}\end{array}\right]$. Since $J()$ is symmetric in its first two arguments, it follows that $O\left(x ; k^{0}\right)$ $=O\left(y ; k^{0}\right)$, so A. 1 satisfies P4. P5' (additivity) follows trivially since A.1 is additive. Similarly, P6' (symmetry in groups with different values of $k$ ), follows from the additive form of A.1. The proof that $O($ ) satisfies $\mathrm{P} 7$ '(a) proceeds in two steps:

Step 1. Since $O\left(\right.$ ) is continuous, assume that contrary to $\mathrm{P} 7^{\prime}$ (a), there exists $\delta$ and $d_{y}^{\prime}$ such that, $O(x ; k)>O(y ; k)$ for $d_{y}=\delta+d_{y}^{\prime}$, and $O(x ; k)=O(y ; k)$ for $d_{y}=\delta$ where $\delta \geq 0, d_{y}^{\prime}>0, \delta+d_{y}^{\prime} \leq x_{1 \mathrm{f}}$, and $d_{y}^{\prime} \in \eta$ with $\eta$ an arbitrary small neighborhood of zero. Let $x^{\prime}$ denote the distribution obtained from $x$ through a movement of $\delta$ type 1 people from group $\mathrm{f}$ to group g, and let $y^{\prime}$ denote the distribution obtained from $x^{\prime}$ through a movement of $d_{y}^{\prime}$ type 1 people from group $\mathrm{f}$ to $\mathrm{g}$. Since $O(x ; k)>O(y ; k)$ for $d_{\mathrm{y}}=\delta+d_{y}^{\prime}$, it must be that $O\left(x^{\prime} ; k\right)>O\left(y^{\prime} ; k\right)$. Then since $O\left(\right.$ ) is differentiable, $\operatorname{Lim}_{d_{y}^{\prime} \rightarrow 0} \frac{\left(O\left(y^{\prime} ; k\right)-O\left(x^{\prime} ; k\right)\right)}{d_{y}^{\prime}}=\partial O\left(y^{\prime} ; k\right) / \partial d_{y}^{\prime}<0$, and from Lemma 1a it follows that,

$$
\partial O\left(y^{\prime} ; k\right) / \partial d_{y}^{\prime}=\frac{\Gamma^{\prime}\{\}_{1}}{N_{1}} \int_{s_{1 f}^{\prime}}^{s_{1}^{\prime}}\left[\partial^{2} J\left(\xi, s_{2 f} ; k_{f}\right) / \partial \xi^{2}\right] d \xi<0,
$$

where $s_{1 f}^{\prime}=s_{1 f}-\delta / N_{1}$ and $s_{1 g}^{\prime}=s_{1 g}+\delta / N_{1}$. Then there must be a value of $\xi$ such that $\partial^{2} J() / \partial \xi^{2}<0$. But that contradicts Theorem 1(e) whereby $\partial^{2} J() / \partial s_{1 j}^{2} \geq 0$. Then as stipulated in $\mathrm{P}^{\prime}(\mathrm{a}), O(x ; k) \leq O(y ; k)$ for all $0 \leq d_{y} \leq x_{1 \mathrm{f}}$.

Step 2. Since $O\left(\right.$ ) is continuous, assume that contrary to $\mathrm{P} 7^{\prime}(\mathrm{a})$, there exists some $\delta$ and $d_{z}^{\prime}=d_{y}^{\prime}$ such that,

$$
\begin{aligned}
& O(y ; k)>O(z ; k) \text { for } d_{\mathrm{y}}=\delta+d_{y}^{\prime}=\delta+d_{z}^{\prime}=d_{\mathrm{z}}, \text { and } \\
& O(y ; k)=O(z ; k) \text { for } d_{\mathrm{y}}=\delta=d_{\mathrm{z}},
\end{aligned}
$$

where $\delta \geq 0, d_{y}^{\prime}>0, \delta+d_{y}^{\prime} \leq x_{1 \mathrm{f}}$, and $d_{y}^{\prime} \in \eta$ with $\eta$ an arbitrary small neighborhood of zero.

Let the $x$ ' and $y$ ' distributions be obtained from $x$ through movements from group $\mathrm{f}$ to group $\mathrm{g}$ in the same way as in step 1 above, and let the $z^{\prime}$ distribution be obtained from $x^{\prime}$ through a movement of $d_{z}^{\prime}$ type 1 people from group $\mathrm{f}$ to group h. Since $O(y ; k)>O(z ; k)$ for $d_{\mathrm{y}}=\delta+d_{y}^{\prime}=\delta+d_{z}^{\prime}=d_{z}$, it must be that $O\left(y^{\prime} ; k\right)>O\left(z^{\prime} ; k\right)$. Then since $O()$ is differentiable, $\partial O\left(z^{\prime} ; k\right) / \partial d_{z}^{\prime}-\partial O\left(y^{\prime} ; k\right) / \partial d_{y}^{\prime}<0$, and from Lemma $1 \mathrm{~b}$ it follows that

$$
\partial O\left(z^{\prime} ; k\right) / \partial d_{z}^{\prime}-\partial O\left(y^{\prime} ; k\right) / \partial d_{y}^{\prime}=-\frac{\Gamma^{\prime}\{\}}{N_{1}} \int_{k_{h}}^{k_{g}}\left[\partial^{2} J\left(s_{1 g}^{\prime}, s_{2 g} ; k\right) / \partial s_{1 g}^{\prime} \partial k\right] d k<0,
$$


where $s_{1 g}^{\prime}=s_{1 g}+\delta / N_{1}$. Then there must be a value of $k$ such that $\partial^{2} J\left(s_{1 g}^{\prime}, s_{2 g} ; k\right) / \partial s_{1 g}^{\prime} \partial k>0$. But that contradicts Theorem 1(c) whereby $\partial^{2} J() / \partial s_{1 g} \partial k_{j} \leq 0$. Then as stipulated in P7'(a) $O(y ; k) \leq O(z ; k)$ for all $0 \leq d_{\mathrm{y}}=d_{\mathrm{z}} \leq x_{1 f} . \mathrm{P}^{\prime}(\mathrm{b})$ is proved in an identical manner except that the step 1 contradiction pertains to the second part of Theorem 1(e), i.e., $\partial^{2} J() / \partial s_{1 j}^{2}=0$, if $s_{2 j}=0$.

The proof that $O($ ) satisfies P8'(a) proceeds in the same way as that for P7'(a). Since $O($ ) is continuous, assume that contrary to $\mathrm{P} 8$ '(a), there exists some $\delta$ and $d^{\prime}{ }_{v}=d_{y}^{\prime}$ such that,

$$
\begin{aligned}
& O(y ; k)>O(\mathrm{v} ; k) \text { for } d_{\mathrm{y}}=\delta+d_{y}^{\prime}=\delta+d_{v}^{\prime}=d_{\mathrm{v}}, \text { and } \\
& O(y ; k)=O(\mathrm{v} ; k) \text { for } d_{\mathrm{y}}=\delta=d_{\mathrm{v}},
\end{aligned}
$$

where $\delta \geq 0, d_{y}^{\prime}>0, \delta+d_{y}^{\prime} \leq x_{1 \mathrm{f}}$, and $d_{y}^{\prime} \in \eta$ with $\eta$ an arbitrary small neighborhood of zero. Let the $x^{\prime}$ and $y^{\prime}$ distributions be obtained from $x$ through movements from group $\mathrm{f}$ to group $\mathrm{g}$ in the same way as in step 1 of the above necessity proof that $O()$ satisfies P7'(a). Let $v^{\prime}$ denote the distribution obtained from $x^{\prime}$ through a movement of $d^{\prime}{ }_{v}$ type 1 people from group q to group $\mathrm{r}$. Since $O(y ; k)>O(v ; k)$ for $d_{\mathrm{y}}=\delta+d_{y}^{\prime}=\delta+d_{v}^{\prime}=d_{v}$, it must be that $O\left(y^{\prime} ; k\right)>O\left(v^{\prime} ; k\right)$. Then since $O\left(\right.$ ) is differentiable, $\partial O\left(v^{\prime} ; k\right) / \partial d_{z}^{\prime}-\partial O\left(y^{\prime} ; k\right) / \partial d_{y}^{\prime}<0$, and from Lemma $1 \mathrm{c}$ it follows that ,

$$
\partial O\left(v^{\prime} ; k\right) / \partial d_{z}^{\prime}-\partial O\left(y^{\prime} ; k\right) / \partial d_{y}^{\prime}=\frac{\Gamma^{\prime}\{\}_{1}}{N_{1}} \int_{k_{g}}^{k_{r}} \int_{s_{1 f}^{\prime}}^{s_{1}^{\prime}}\left[\partial^{3} J\left(\xi, s_{2 g} ; k\right) / \partial \xi^{2} \partial k\right] d \xi d k<0
$$

where $s_{1 f}^{\prime}=s_{1 f}-\delta / N_{1}$ and $s_{1 g}^{\prime}=s_{1 g}+\delta / N_{1}$. Then there must be some $\xi$ and $k$ such that $\partial^{3} J\left(\xi, s_{2 g} ; k\right) / \partial \xi^{2} \partial k<0$. But that contradicts Theorem 1(d) whereby $\partial^{3} J() / \partial s_{1 j}^{2} \partial k_{j} \geq 0$. Then as stipulated in $\mathrm{P} 8$ ' (a) $O(y ; k) \leq O(v ; k)$ for all $0 \leq d_{\mathrm{y}}=d_{\mathrm{v}} \leq x_{\mathrm{ff}}$. Since from the above necessity proof for $\mathrm{P} 7$ '(a) $O(x ; k) \leq O(y ; k)$ for all $0 \leq d_{\mathrm{y}} \leq x_{1 \mathrm{f}}$, it follows that $O(v ; k) \geq O(y ; k) \geq$ $O(x ; k)$ for all $0 \leq d_{\mathrm{y}}=d_{\mathrm{v}} \leq x_{1 \mathrm{f}}$. P8'(b) is proved in an identical manner except that the contradiction pertains to the second part of Theorem 1(d), i.e., $\partial^{3} J() / \partial s_{1 j}^{2} \partial k_{j}=0$ if $s_{2 j}=0$. This completes the proof of necessity and thereby the proof of Theorem 1 .

\section{Proof of Corollary 1.1}

Sufficiency: If $O(x ; k)$ satisfies properties 1-4, 5', 6' - 8', then it takes the form of (6) in the text.

Sufficiency is proved in four steps.

Step 1.If $\mathrm{O}\left(\mathrm{x} ; k^{0}\right)$ not only satisfies properties $1-4,6^{\prime}-8^{\prime}$ but also P5", and $k^{0}$ is a vector with $k_{1}$ $=k_{2}=\ldots=k_{\mathrm{T}}=\tilde{k}$ then,

$O\left(x ; k^{0}\right)=\Gamma\left\{\sum_{j=1}^{T} J\left(s_{1 j}, s_{2 j} ; \tilde{k}\right)\right\}=\gamma_{0}(\tilde{k})+\gamma_{1}(\tilde{k})\left(\sum_{j=1}^{T} J\left(s_{1 j}, s_{2 j} ; \tilde{k}\right)\right)=b\left(1-\sum_{j=1}^{T} \sqrt{s_{1 j} s_{2 j}}\right)$

where $\mathrm{b}$ is positive and $\gamma_{0}(\tilde{k})$ and $\gamma_{1}(\tilde{k})$ are parameters with $\gamma_{0}(\tilde{k})=b-\gamma_{1}(\tilde{k}) 2 \lambda(\tilde{k})$.

Hutchens (2004) defines a measure of segregation as a member of the RIMFO class if it exhibits properties $\mathrm{P} 1, \mathrm{P} 2, \mathrm{P} 6$ and $\mathrm{P} 7$ above. Then from Corollary $1.2, O\left(x ; k^{0}\right)$ belongs to the 
RIMFO class. Moreover, given that $O\left(x ; k^{0}\right)$ satisfies P5 ${ }^{\prime}, O\left(x ; k^{0}\right)$ is an additive decomposable RIMFO. According to the corollary to Theorem 1 in Hutchens (2004), a segregation index $O(x)$ is an additive decomposable RIMFO if and only if $O(x)$ is a positive multiple of $O_{\mathrm{c}}(x), 0<c<1$, $\mathrm{x} \in D$, where

$$
O_{c}(x)=-\sum_{j=1}^{T} s_{2 j}\left[\left(s_{1 j} / s_{2 j}\right)^{c}-1\right]=\left(1-\sum_{j=1}^{T} s_{1 j}^{c} s_{2 j}^{1-c}\right) .
$$

Then

$$
O\left(x ; k^{0}\right)=\Gamma\left\{\sum_{j=1}^{T} J\left(s_{1 j}, s_{2 j} ; \tilde{k}\right)\right\}=b\left(1-\sum_{j=1}^{T} s_{1 j}^{c} s_{2 j}^{1-c}\right) .
$$

Let $O\left(y ; k^{0}\right)$ be an index that is identical to $O\left(x ; k^{0}\right)$ except that type 2 people are labeled type 1 and type 1 people labeled type 2 . Thus,

$$
O\left(y ; k^{0}\right)=\Gamma\left\{\sum_{j=1}^{T} J\left(s_{2 j}, s_{1 j} ; \tilde{k}\right)\right\}=b\left(1-\sum_{j=1}^{T} s_{2 j}^{c} s_{1 j}^{1-c}\right) .
$$

Since $O\left(x ; k^{0}\right)$ satisfies symmetry in types (P4), for any $\mathrm{x} \in D$ it follows that,

$$
0=O\left(x ; k^{0}\right)-O\left(y ; k^{0}\right)=b\left(1-\sum_{j=1}^{T} s_{1 j}^{c} s_{2 j}^{1-c}\right)-b\left(1-\sum_{j=1}^{T} s_{2 j}^{c} s_{1 j}^{1-c}\right)=b \sum_{j=1}^{T} \sqrt{s_{1 j} s_{2 j}}\left[\left(s_{2 j} / s_{1 j}\right)^{c-1 / 2}-\left(s_{1 j} / s_{2 j}\right)^{c-1 / 2}\right]
$$

Since this is true for any $x \in D$, the expression in brackets is zero for any $x \in D$. Then $c=1 / 2$ and,

$$
O\left(x ; k^{0}\right)=\Gamma\left\{\sum_{j=1}^{T} J\left(s_{1 j}, s_{2 j} ; \tilde{k}\right)\right\}=b\left(1-\sum_{j=1}^{T} \sqrt{s_{1 j} s_{2 j}}\right) .
$$

To show that,

$$
\Gamma\left\{\sum_{j=1}^{T} J\left(s_{1 j}, s_{2 j} ; \tilde{k}\right)\right\}=\gamma_{0}(\tilde{k})+\gamma_{1}(\tilde{k})\left(\sum_{j=1}^{T} J\left(s_{1 j}, s_{2 j} ; \tilde{k}\right)\right)
$$

let there be three groups $(T=3)$ such that,

\begin{tabular}{|c|c|c|c|}
\hline $\begin{array}{c}\text { Group } \\
\text { Number }\end{array}$ & $\begin{array}{c}\text { Share of type } \\
1 \text { people }\left(s_{1 \mathrm{j}}\right)\end{array}$ & $\begin{array}{c}\text { Share of type } \\
2 \text { people }\left(s_{2 \mathrm{j}}\right)\end{array}$ & $J\left(s_{1 j}, s_{2 j} ; \tilde{k}\right)=L\left(s_{1 j}, s_{2 j} ; \tilde{k}\right)+\lambda(\tilde{k})\left(s_{1 j}+s_{2 j}\right)$ \\
\hline 1 & $1-\theta$ & 0 & $J(1-\theta, 0 ; \tilde{k})=L(1-\theta, 0 ; \tilde{k})+\lambda(\tilde{k})(1-\theta)$ \\
\hline 2 & 0 & $1-\theta$ & $J(0,1-\theta ; \tilde{k})=L(0,1-\theta ; \tilde{k})+\lambda(\tilde{k})(1-\theta)$ \\
\hline 3 & $\theta$ & $\theta$ & $J(\theta, \theta ; \tilde{k})=L(\theta, \theta ; \tilde{k})+\lambda(\tilde{k})(2 \theta)$ \\
\hline
\end{tabular}

From (a) of Theorem $1, L(1-\theta, 0 ; \tilde{k})=L(0,1-\theta ; \tilde{k})=0$, and from (b) of Theorem $1, L(\mathrm{)}$ is homogeneous of degree 1 in its first two argument implying $L(\theta, \theta ; \tilde{k})=\theta L(1,1 ; \tilde{k})$. Then for these three groups, equation (A.8) yields,

(A.9) $\quad \Gamma\{\theta L(1,1 ; \tilde{k})+2 \lambda(\tilde{k})\}=b(1-\theta)$

Note that (A.9) implies $\Gamma\{2 \lambda(\tilde{k})\}=b$ and $\Gamma\{L(1,1 ; \tilde{k})+2 \lambda(\tilde{k})\}=0$. Then (A.9) can be equivalently written, 


$$
\begin{aligned}
\Gamma\{(1-\theta) 2 \lambda(\tilde{k})+\theta(L(1,1 ; \tilde{k})+2 \lambda(\tilde{k}))\} & =(1-\theta) b+\theta(0) \\
& =(1-\theta) \Gamma\{2 \lambda(\tilde{k})\}+\theta(\Gamma\{L(1,1 ; \tilde{k})+2 \lambda(\tilde{k})\})
\end{aligned}
$$

Then $\Gamma\{\}$ is affine. As such an equivalent way to write equation (A.8) is,

$$
O\left(x ; k^{0}\right)=\Gamma\left\{\sum_{j=1}^{T} J\left(s_{1 j}, s_{2 j} ; \tilde{k}\right)\right\}=\gamma_{0}(\tilde{k})+\gamma_{1}(\tilde{k})\left(\sum_{j=1}^{T} J\left(s_{1 j}, s_{2 j} ; \tilde{k}\right)\right)=b\left(1-\sum_{j=1}^{T} \sqrt{s_{1 j} s_{2 j}}\right)
$$

where $b$ is positive and $\gamma_{0}(\tilde{k})$ and $\gamma_{1}(\tilde{k})$ are parameters. Moreover, since $\Gamma\{2 \lambda(\tilde{k})\}=b$, it follows that $\gamma_{0}(\tilde{k})+\gamma_{1}(\tilde{k}) 2 \lambda(\tilde{k})=b$. Then $\gamma_{0}(\tilde{k})=b-\gamma_{1}(\tilde{k}) 2 \lambda(\tilde{k})$.

Step 2.

$$
J\left(s_{1 j}, s_{2 j} ; \tilde{k}\right)=\left[\lambda(\tilde{k})\left(s_{1 j}+s_{2 j}\right)-\frac{b}{\gamma_{1}(\tilde{k})} \sqrt{s_{1 j} s_{2 j}}\right]
$$

Note that from Step 1,

$$
\gamma_{0}(\tilde{k})+\gamma_{1}(\tilde{k})\left(\sum_{j=1}^{T} J\left(s_{1 j}, s_{2 j} ; \tilde{k}\right)\right)=b\left(1-\sum_{j=1}^{T} \sqrt{s_{1 j} s_{2 j}}\right) .
$$

Substitute into that

$$
\gamma_{0}(\tilde{k})=b-\gamma_{1}(\tilde{k}) 2 \lambda(\tilde{k}) \text { and } J\left(s_{1 j}, s_{2 j} ; \tilde{k}\right)=L\left(s_{1 j}, s_{2 j} ; \tilde{k}\right)+\lambda(\tilde{k})\left(s_{1 j}+s_{2 j}\right),
$$

and noting that,

$$
\sum_{j=1}^{T} \lambda(\tilde{k})\left(s_{1 j}+s_{2 j}\right)=2 \lambda(\tilde{k})
$$

obtain,

$$
\text { (A.10) } \quad \gamma_{1}(\tilde{k})\left[\sum_{j=1}^{T} L\left(s_{1 j}, s_{2 j} ; \tilde{k}\right)\right]=-b \sum_{j=1}^{T} \sqrt{s_{1 j} s_{2 j}} .
$$

In a manner similar to Step 1 , let there be three groups $(T=3)$ of the form,

\begin{tabular}{|c|c|c|c|}
\hline $\begin{array}{l}\text { Group } \\
\text { Number }\end{array}$ & $\begin{array}{l}\text { Share of type 1 } \\
\text { people }\left(s_{1 \mathrm{j}}\right)\end{array}$ & $\begin{array}{l}\text { Share of type 2 } \\
\text { people }\left(s_{2 \mathrm{j}}\right)\end{array}$ & $L\left(s_{1 j}, s_{2 j} ; \tilde{k}\right)$ \\
\hline 1 & $1-\alpha$ & 0 & $L(1-\alpha, 0 ; \tilde{k})=0$ \\
\hline 2 & 0 & $1-\beta$ & $L(0,1-\beta ; \tilde{k})=0$ \\
\hline 3 & $\alpha$ & $\beta$ & $L(\alpha, \beta ; \tilde{k})$ \\
\hline
\end{tabular}

where $0 \leq \alpha \leq 1$ and $0 \leq \beta \leq 1$, and the last column is from (a) of Theorem 1. Substituting the three groups into A.10, $\gamma_{1}(\tilde{k}) L(\alpha, \beta ; \tilde{k})=-b \sqrt{\alpha \beta}$ or equivalently, $L(\alpha, \beta ; \tilde{k})=\frac{-b}{\gamma_{1}(\tilde{k})} \sqrt{\alpha \beta}$. Then since $J\left(s_{1 j}, s_{2 j} ; \tilde{k}\right)=L\left(s_{1 j}, s_{2 j} ; \tilde{k}\right)+\lambda(\tilde{k})\left(s_{1 j}+s_{2 j}\right)$, it follows that, 


$$
J\left(s_{1 j}, s_{2 j} ; \tilde{k}\right)=\left[\lambda(\tilde{k})\left(s_{1 j}+s_{2 j}\right)-\frac{b}{\gamma_{1}(\tilde{k})} \sqrt{s_{1 j} s_{2 j}}\right] .
$$

Step 3. For any feasible value of $k_{\mathrm{j}}$,

$$
J\left(s_{1 j}, s_{2 j} ; k_{j}\right)=\left[\lambda\left(k_{j}\right)\left(s_{1 j}+s_{2 j}\right)-\frac{b}{\gamma_{1}\left(k_{j}\right)} \sqrt{s_{1 j} s_{2 j}}\right] .
$$

Recall that $\tilde{k}$ is a non-negative number and that $k_{1}=k_{2}=\ldots=k_{\mathrm{T}}=\tilde{k}$. Designate a new $k$ vector as $k^{\prime}$ where $k_{T}^{\prime}=\tilde{k}$ and the $k_{j}^{\prime} \leq \tilde{>} \tilde{k}, \mathrm{j}=1, \ldots, \mathrm{T}-1$. Since $k_{T}^{\prime}=\tilde{k}$, it follows that

$$
J\left(s_{1 T}, s_{2 T} ; k_{T}^{\prime}\right)=J\left(s_{1 T}, s_{2 T} ; \tilde{k}\right)=\left[\lambda(\tilde{k})\left(s_{1 T}+s_{2 T}\right)-\frac{b}{\gamma_{1}(\tilde{k})} \sqrt{s_{1 T} s_{2 T}}\right]
$$

for all feasible values of $k_{j}^{\prime}, \mathrm{j}=1, \mathrm{~T}-1$. Since $\tilde{k}$ can be any non-negative number and since the corollary requires that the index is additive in groups (P5',) and symmetric in groups (P6'), it follows that for all feasible values of the vector $k$,

$$
J\left(s_{1 j}, s_{2 j} ; k_{j}\right)=\left[\lambda\left(k_{j}\right)\left(s_{1 j}+s_{2 j}\right)-\frac{b}{\gamma_{1}\left(k_{j}\right)} \sqrt{s_{1 j} s_{2 j}}\right] .
$$

Step 4. Substitute the above expression for $J\left(s_{1 \mathrm{j}}, s_{2 \mathrm{j}} ; k_{\mathrm{j}}\right)$ as well as $\gamma_{0}(\tilde{k})=b-\gamma_{1}(\tilde{k}) 2 \lambda(\tilde{k})$ into

$$
O(x ; k)=\Gamma\left\{\sum_{j=1}^{T} J\left(s_{1 j}, s_{2 j} ; k_{j}\right)\right\}=\gamma_{0}(\tilde{k})+\gamma_{1}(\tilde{k})\left(\sum_{j=1}^{T} J\left(s_{1 j}, s_{2 j} ; k_{j}\right)\right)
$$

to obtain,

$$
O(x ; k)=b-\gamma_{1}(\tilde{k}) 2 \lambda(\tilde{k})+\gamma_{1}(\tilde{k}) \sum_{j=1}^{T}\left[\lambda\left(k^{j}\right)\left(s_{1 j}+s_{2 j}\right)-\frac{b}{\gamma_{1}\left(k^{j}\right)} \sqrt{s_{1 j} s_{2 j}}\right]
$$

This completes the proof of Corollary 1.1. Dropping the subscript on $\gamma$ (thus, letting $\gamma()=\gamma_{1}()$ ) yields the expression in the text.

Necessity: If $O(x ; k)$ is of the form of $O_{\mathrm{b}}(x ; k)$ (equation (6) in the text), then $O(x ; k)$ satisfies properties $1-4,5$ ', 6 ' - 8'.

$O_{\mathrm{b}}(x ; k)$ satisfies properties $1-4,5^{\prime}-8^{\prime}$ because it meets the conditions of that Theorem 1 . To see this, rewrite $\mathrm{O}_{\mathrm{b}}(x ; k)$ in the form of (A.1).

$$
\begin{aligned}
& O_{b}(x ; k)=\Gamma\left\{\sum_{j=1}^{T} J\left(s_{1 j}, s_{2 j} ; k_{j}\right)\right\} \text { with } \\
& \Gamma\{a\}=b-\gamma_{1}(\tilde{k}) 2 \lambda(\tilde{k})+\gamma_{1}(\tilde{k}) a \text { and } J\left(s_{1 j}, s_{2 j} ; k_{j}\right)=\lambda\left(k_{j}\right)\left(s_{1 j}+s_{2 j}\right)-\frac{b}{\gamma_{1}\left(k_{j}\right)} \sqrt{s_{1 j} s_{2 j}}
\end{aligned}
$$


where $\gamma\left(k_{\mathrm{j}}\right)$ is a positive non-increasing differentiable function of $k_{\mathrm{j}}$, and $\lambda\left(k_{\mathrm{j}}\right)$ is a non-increasing differentiable function of $\mathrm{k}_{\mathrm{j}}$. By implication $\Gamma\{\}$ is a strictly increasing differentiable function of a. Condition (a) and (b) of Theorem 1 are obviously satisfied. Furthermore,

$$
\begin{gathered}
\text { c) } \partial^{2} J() / \partial s_{1 j} \partial k_{j}=\frac{b s_{2 j}{ }^{1 / 2} s_{1 j}{ }^{-1 / 2}}{2\left[\gamma_{1}\left(k_{j}\right)\right]^{2}}\left[\partial \gamma_{1}\left(k_{j}\right) / \partial k_{j}\right] \leq 0 \text { and } \\
\partial^{2} J() / \partial s_{2 j} \partial k_{j}=\frac{b s_{2 j}{ }^{-1 / 2} s_{1 j}{ }^{1 / 2}}{2\left[\gamma_{1}\left(k_{j}\right)\right]^{2}}\left[\partial \gamma_{1}\left(k_{j}\right) / \partial k_{j}\right] \leq 0, \mathrm{j}=1, \ldots, \mathrm{T}, \\
\begin{array}{c}
\partial^{3} J() / \partial s_{1 j}^{2} \partial k_{j}=\frac{-b s_{2 j}{ }^{1 / 2} s_{1 j}{ }^{-3 / 2}}{4\left[\gamma_{1}\left(k_{j}\right)\right]^{2}}\left[\partial \gamma_{1}\left(k_{j}\right) / \partial k_{j}\right] \geq 0 \text { if } s_{2 j}>0 \\
\text { e) } \partial^{2} J() / \partial s_{1 j}^{2}=\frac{b s_{2 j}{ }^{1 / 2} s_{1 j}{ }^{-3 / 2}}{4\left[\gamma_{1}\left(k_{j}\right)\right]} \geq 0 \text { if } s_{2 j}>0 \\
\text { and } \partial^{2} J() / \partial s_{1 j}^{2}=0 \text { if } s_{2 j}=0, \mathrm{j}=1, \ldots, \mathrm{T}, \\
\text { f) } \partial^{3} J() / \partial s_{2 j}^{2} \partial k_{j}=\frac{-b s_{1 j}{ }^{1 / 2} s_{2 j}{ }^{-3 / 2}}{4\left[\gamma_{1}\left(k_{j}\right)\right]^{2}}\left[\partial \gamma_{1}\left(k_{j}\right) / \partial k_{j}\right] \geq 0 \text { if } s_{1 j}>0 \\
\text { and } \partial^{3} J() / \partial s_{2 j}^{2} \partial k_{j}=0 \text { if } s_{1 j}=0, \mathrm{j}=1, \ldots, \mathrm{T}, \\
\text { g) } \partial^{2} J() / \partial s_{2 j}^{2}=\frac{b s_{1 j}{ }^{1 / 2} s_{2 j}{ }^{-3 / 2}}{4\left[\gamma_{1}\left(k_{j}\right)\right]} \geq 0 \text { if } s_{1 j}>0 \\
\text { and } \partial^{2} J() / \partial s_{2 j}^{2}=0 \text { if } s_{1 j}=0, \mathrm{j}=1, \ldots, \mathrm{T} \text {. }
\end{array}
\end{gathered}
$$

Moreover, $O_{b}(x ; k)$ is additive decomposable for groups with the same economic status. To see this, let all groups have the same economic status such that $k_{\mathrm{j}}=\tilde{k}, \mathrm{j}=1, \ldots, \mathrm{T}$, where $\tilde{k}$ is a nonnegative number. Then,

$$
\begin{aligned}
O_{b}(x ; k) & =b-\gamma_{1}(\tilde{k}) 2 \lambda(\tilde{k})+\gamma_{1}(\tilde{k}) \sum_{j=1}^{T}\left[\lambda(\tilde{k})\left(s_{1 j}+s_{2 j}\right)-\frac{b}{\gamma_{1}(\tilde{k})} \sqrt{s_{1 j} s_{2 j}}\right] \\
& =b-\gamma_{1}(\tilde{k}) 2 \lambda(\tilde{k})+\gamma_{1}(\tilde{k}) 2 \lambda(\tilde{k})-\gamma_{1}(\tilde{k}) \sum_{j=1}^{T} \frac{b}{\gamma_{1}(\tilde{k})} \sqrt{s_{1 j} s_{2 j}} \\
& =b\left[1-\sum_{j=1}^{T} \sqrt{s_{1 j} s_{2 j}}\right]
\end{aligned}
$$

Thus, when all groups have the same economics status, $O_{b}(x ; k)$ is a positive multiple of the square root index. Theorem 2 in Hutchens (2004) establishes that the square root index is additive decomposable (in particular, see Lemma A.1). Since a positive multiple of an additive decomposable measure of segregation is also additive decomposable, $O_{b}(x ; k)$ is additive 
decomposable when all groups have the same economic status. This then completes the proof of necessity.

\section{Proof of Corollary 1.2}

Given $k_{1}^{0}=k_{2}^{0}=\ldots=k_{T}^{0}$, from Theorem 1 if $O(x ; k)$ is of the form of (A.1) then $O(x ; k)$ satisfies properties 1- 4. P5 follows trivially since (A.1) is additive and $J()$ is 3-smooth and thereby differentiable. Similarly P6 follows from the additive form of (A.1). For P7, note from Theorem 1(e) $\partial^{2} J() / \partial s_{1 j}^{2} \geq 0$ if $s_{2 j}>0$. Then from Berge (1997), p. 199, $J()$ is a convex function for all $s_{1 j} \geq$ and $s_{2 j}>0$. Let $y$ be obtained from $x$ by a disequalizing movement from group $f$ to group $g$. Then,

$$
O\left(y ; k^{0}\right)=\Gamma\left\{\sum_{h \neq f, g} J\left(s_{1 h}, s_{2 h} ; k_{h}^{0}\right)+J\left(s_{1 f}-\frac{d}{N_{1}}, s_{2 f} ; k_{f}^{0}\right)+J\left(s_{1 g}+\frac{d}{N_{1}}, s_{2 g} ; k_{g}^{0}\right)\right\}
$$

where $\mathrm{s}_{2 f}=s_{2 g}>0, s_{1 f}<s_{1 g}$ and $0<d \leq x_{1 f}$. Since $J$ is convex, and $\Gamma$ is strictly increasing, we have

$$
O\left(y ; k^{0}\right) \geq \Gamma\left\{\sum_{h \neq f, g} J\left(s_{1 h}, s_{2 h} ; k_{h}^{0}\right)+J\left(s_{1 f}, s_{2 f} ; k_{f}^{0}\right)+J\left(s_{1 g}, s_{2 g} ; k_{g}^{0}\right)\right\}=O\left(x ; k^{0}\right)
$$

which establishes P7.

Two lemmas are useful in proving Theorem 2.

\section{Lemma 2.}

$$
\begin{gathered}
\sum_{j=1}^{T_{g}}\left[L\left(s_{1 j g}, s_{2 j g} ; k_{g}^{*}\right)+\lambda\left(k_{g}^{*}\right)\left(s_{1 j g}+s_{2 j g}\right)\right]=\lambda\left(k_{g}^{*}\right) \sum_{j=1}^{T_{g}}\left(s_{1 j g}+s_{2 j g}\right)+\left[\sum_{j=1}^{T_{g}} s_{2 j g}\right]\left[\partial L\left(\hat{q}, 1 ; k_{g}^{*}\right) / \partial q\right]\left[\hat{q}-\varphi_{g}(\hat{q})\right] \\
-\left[\sum_{j=1}^{T_{g}} s_{2 j g}\right] \int_{0}^{\hat{q}}\left[\partial^{2} L\left(q, 1 ; k_{g}^{*}\right) / \partial q^{2}\right]\left[q-\varphi_{g}(q)\right] d q
\end{gathered}
$$

\section{Proof of Lemma 2:}

Since $L()$ is homogeneous of degree 1 in its first two arguments and $L\left(0, s_{2 j} ; k_{g}^{*}\right)=0$, it follows that,

$$
L\left(s_{1 j g}, s_{2 j g} ; k_{g}^{*}\right)=s_{2 j g} L\left(q_{j g}, 1 ; k_{g}^{*}\right)=s_{2 j g} \int_{0}^{q_{j g}} \partial L\left(q, 1 ; k_{g}^{*}\right) / \partial q d q=s_{2 j g} \int_{0}^{\hat{q}}\left[\partial L\left(q, 1 ; k_{g}^{*}\right) / \partial q\right]\left[1-\rho\left(q, q_{j g}\right)\right] d q
$$

where $q_{j g}=s_{1 j g} / s_{2 j g}$. Then,

$$
\left.\sum_{j=1}^{T_{g}} L\left(s_{1 j g}, s_{2 j g} ; k_{g}^{*}\right)=\int_{0}^{\hat{q}}\left[\partial L\left(q, 1 ; k_{g}^{*}\right) / \partial q\right]\left[\sum_{j=1}^{T_{g}} s_{2 j g}\left(1-\rho\left(q, q_{j g}\right)\right)\right] d q=\left[\sum_{j=1}^{T_{g}} s_{2 j g}\right]\right]_{0}^{\hat{q}}\left[\partial L\left(q, 1 ; k_{g}^{*}\right) / \partial q\right][1-F(q)] d q
$$

Integrating by parts,

$$
\begin{aligned}
\sum_{j=1}^{T_{g}} L\left(s_{1 j g}, s_{2 j g} ; k_{g}^{*}\right)= & {\left[\sum_{j=1}^{T_{g}} s_{2 j g}\right]\left[\left(q-\varphi_{g}(q)\right) \partial L\left(q, 1 ; k_{g}^{*}\right) / \partial q\right]_{0}^{\hat{q}} } \\
& \left.-\left[\sum_{j=1}^{T_{g}} s_{2 j g}\right]\right]_{0}^{\hat{q}}\left[\partial^{2} L\left(q, 1 ; k_{g}^{*}\right) / \partial q^{2}\right]\left[q-\varphi_{g}(q)\right] d q
\end{aligned}
$$


Since $\varphi_{g}(0)=0$, it follows that,

$$
\left.\sum_{j=1}^{T_{g}} L\left(s_{1 j g}, s_{2 j g} ; k_{g}^{*}\right)=\left[\partial L\left(\hat{q}, 1 ; k_{g}^{*}\right) / \partial q\right]\left[\hat{q}-\varphi_{g}(\hat{q})\right] \sum_{j=1}^{T_{g}} s_{2 j g}-\left[\sum_{j=1}^{T_{g}} s_{2 j g}\right]\right]_{0}^{\hat{q}}\left[\partial^{2} L\left(q, 1 ; k_{g}^{*}\right) / \partial q^{2}\right]\left[q-\varphi_{g}(q)\right] d q .
$$

Then $\sum_{j=1}^{T_{g}}\left[L\left(s_{1 j g}, s_{2 j g} ; k_{g}^{*}\right)+\lambda\left(k_{g}^{*}\right)\left(s_{1 j g}+s_{2 j g}\right)\right]=\lambda\left(k_{g}\right) \sum_{j=1}^{T_{g}}\left(s_{1 j g}+s_{2 j g}\right)$

$$
\left.+\left[\sum_{j=1}^{T_{g}} s_{2 j g}\right]\left[\partial L\left(\hat{q}, 1 ; k_{g}^{*}\right) / \partial q\right]\left[\hat{q}-\varphi_{g}(\hat{q})\right]-\left[\sum_{j=1}^{T_{g}} s_{2 j g}\right]\right]_{0}^{\hat{q}}\left[\partial^{2} L\left(q, 1 ; k_{g}^{*}\right) / \partial q^{2}\right]\left[q-\varphi_{g}(q)\right] d q
$$

Lemma 3.
(a) $\varphi_{g}(\hat{q})=\hat{q}-\sum_{j=1}^{T_{g}} s_{1 j g} / \sum_{j=1}^{T_{g}} s_{2 j g}$
(b) $C_{g}(\hat{q})=\sum_{j=1}^{T_{g}} s_{1 j g}^{x}-\sum_{j=1}^{T_{g}} s_{1 j g}^{y}=B_{1 g}$

\section{Proof of Lemma 3:}

To establish (a) note that,

$$
\begin{gathered}
\varphi_{g}(\hat{q})=\int_{0}^{\hat{q}} F(q) d q=\sum_{j=1}^{T_{g}} s_{2 j g} \max \left[0, \hat{q}-q_{j g}\right] / \sum_{j=1}^{T_{g}} s_{2 j g}=\sum_{j=1}^{T_{g}} s_{2 j g}\left[\hat{q}-q_{j g}\right] / \sum_{j=1}^{T_{g}} s_{2 j g}= \\
{\left[\begin{array}{c}
T_{g} \\
\left.\hat{q} \sum_{j=1}^{g} s_{2 j g}-\sum_{j=1}^{T_{g}} s_{1 j g}\right] / \sum_{j=1}^{T_{g}} s_{2 j g}=\hat{q}-\sum_{j=1}^{T_{g}} s_{1 j g} / \sum_{j=1}^{T_{g}} s_{2 j g}
\end{array}\right.}
\end{gathered}
$$

To establish (b) note that $C_{g}(\hat{q})=\left[\hat{q}-\varphi_{g}^{x}(\hat{q})\right]_{j=1}^{T_{g}^{x}} s_{2 j g}^{x}-\left[\hat{q}-\varphi_{g}^{y}(\hat{q})\right]_{j=1}^{T_{g}^{y}} s_{2 j g}^{y}$. Then substituting $\varphi_{g}^{x}(\hat{q})$ and $\varphi_{g}^{y}(\hat{q})$ from (a), $C_{g}(\hat{q})=\sum_{j=1}^{T_{g}} s_{1 j g}^{x}-\sum_{j=1}^{T_{g}} s_{1 j g}^{y}=B_{1 g}$.

\section{Theorem 2.}

For all measures of segregation $O\left(\right.$ ) satisfying properties $1-4,5^{\prime}-8$ ', and for all measures of prestige $k$ satisfying $k_{1}^{*}>k_{2}^{*}>\ldots>k_{G}^{*} \geq 0$ and $k_{j g}=k_{g}^{*}, j=1, \ldots, T_{g}, g=1, G$, the following conditions are necessary and sufficient for $O(x ; k) \geq O(y ; k)$ :

(A.11.1) $\sum_{g=1}^{\tau} C_{g}(q) \leq 0$ where $C_{g}(q)=\left(q-\varphi_{g}^{x}(q)\right) \sum_{j=1}^{T_{g}^{x}} s_{2 j g}^{x}-\left(q-\varphi_{g}^{y}(q)\right) \sum_{j=1}^{T_{g}^{y}} s_{2 j g}^{y}$

(A.11.2) $\sum_{g=1}^{\tau} B_{2 g} \leq 0$ where $B_{2 g}=\sum_{j=1}^{T_{g}^{x}} s_{2 j g}^{x}-\sum_{j=1}^{T_{g}^{y}} s_{2 j g}^{y}$

for all $q, 0 \leq q \leq \hat{q}$, and $\tau=1, \ldots, \mathrm{G}$. 


\section{Proof of Theorem 2:}

Let $\Delta O=O(x ; k)-O(y ; k)=\Gamma\left\{Z^{x}\right\}-\Gamma\left\{Z^{y}\right\}$, where $\Gamma\{\}$ is a strictly increasing real valued function, and $Z^{x}=\sum_{g=1}^{G} \sum_{j=1}^{T_{g}^{x}} J\left(s_{1 j g}^{x}, s_{2 j g}^{x} ; k_{g}^{*}\right)$ and $Z^{y}=\sum_{g=1}^{G} \sum_{j=1}^{T_{g}^{y}} J\left(s_{1 j g}^{y}, s_{2 j g}^{y} ; k_{g}^{*}\right)$. Then $\Delta O \geq 0$ iff $\Delta Z \geq 0$ where $\Delta Z=Z^{x}-Z^{y}=\sum_{g=1}^{G} \Delta Z_{g}$ and

$$
\begin{aligned}
\Delta Z_{g} & =\sum_{j=1}^{T_{g}^{x}} J\left(s_{1 j g}^{x}, s_{2 j g}^{x} ; k_{g}^{*}\right)-\sum_{j=1}^{T_{g}^{y}} J\left(s_{1 j g}^{y}, s_{2 j g}^{y} ; k_{g}^{*}\right) \\
& =\lambda\left(k_{g}^{*}\right)\left[\sum_{j=1}^{T_{g}^{x}}\left(s_{1 j g}^{x}+s_{2 j g}^{x}\right)-\sum_{j=1}^{T_{g}^{y}}\left(s_{1 j g}^{y}+s_{2 j g}^{y}\right)\right]+\sum_{j=1}^{T_{g}^{x}} L\left(s_{1 j g}^{x}, s_{2 j g}^{x} ; k_{g}^{*}\right)-\sum_{j=1}^{T_{g}^{y}} L\left(s_{1 j g}^{y}, s_{2 j g}^{y} ; k_{g}^{*}\right)
\end{aligned}
$$

This proof thus shows that conditions A.11 (A.11.1 and A.11.2) are necessary and sufficient condition for $\Delta Z \geq 0$.

\section{Sufficiency}

Using Lemma 2 and Lemma 3, write $\Delta \mathrm{Z}_{g}$ as

$$
\Delta Z_{g}=\lambda\left(k_{g}^{*}\right)\left[B_{1 g}+B_{2 g}\right]+C_{g}(\hat{q})\left[\partial L\left(\hat{q}, 1 ; k_{g}^{*}\right) / \partial q\right]-\int_{0}^{\hat{q}}\left[\partial^{2} L\left(q, 1 ; k_{g}^{*}\right) / \partial q^{2}\right] C_{g}(q) d q
$$

where,

$$
\begin{aligned}
& B_{1 g}=\sum_{j=1}^{T_{g}^{x}} s_{1 j g}^{x}-\sum_{j=1}^{T_{g}^{y}} s_{1 j g}^{y}, B_{2 g}=\sum_{j=1}^{T_{g}^{x}} s_{2 j g}^{x}-\sum_{j=1}^{T_{g}^{y}} s_{2 j g}^{y}, \text { and } \\
& C_{g}(q)=\left(q-\varphi_{g}^{x}(q)\right) \sum_{j=1}^{T_{g}^{x}} s_{2 j g}^{x}-\left(q-\varphi_{g}^{y}(q)\right) \sum_{j=1}^{T_{g}^{y}} s_{2 j g}^{y} .
\end{aligned}
$$

Since from Lemma 3(b), $B_{1 g}=C_{g}(\hat{q})$, equation (A.12) can be equivalently written,

$$
\Delta Z_{g}=\alpha_{1 g} C_{g}(\hat{q})+\alpha_{2 g} B_{2 g}-\int_{0}^{\hat{q}}\left[\partial^{2} L\left(q, 1 ; k_{g}^{*}\right) / \partial q^{2}\right] C_{g}(q) d q,
$$

where $\alpha_{1 g}=\lambda\left(k_{g}^{*}\right)+\partial L\left(\hat{q}, 1 ; k_{g}^{*}\right) / \partial q$ and $\alpha_{2 g}=\lambda\left(k_{g}^{*}\right)$.

With regard to $\alpha_{2 g}$, from Theorem $1 \lambda\left(k_{g}^{*}\right)$ is non-increasing in $k_{g}^{*}$. Then $\partial \alpha_{2 g} / \partial k_{g}^{*} \leq 0$, and (since $k_{g-1}^{*}>k_{g}^{*}$ ), $\alpha_{2(g-1)} \leq \alpha_{2 g}, \mathrm{~g}=2, . ., \mathrm{G}$. With regard to $\alpha_{1 g}$, from (c) of Theorem 1 , $\partial L^{2}\left(\hat{q}, 1 ; k_{g}^{*}\right) / \partial q \partial k_{g}+\partial \lambda\left(k_{g}^{*}\right) / \partial k_{g} \leq 0$. Then $\partial \alpha_{1 g} / \partial k_{g}^{*} \leq 0$, and (since $\left.k_{g-1}^{*}>k_{g}^{*}\right), \alpha_{1(g-1)} \leq \alpha_{1 g}, \mathrm{~g}=2, . ., \mathrm{G}$.

Finally, from (d) and (e) of Theorem $1, \partial^{3} L() / \partial s_{1 j}^{2} \partial k_{j} \geq 0$ and $\partial^{2} L() / \partial s_{1 j}^{2} \geq 0$, and (since $k_{g-1}^{*}>k_{g}^{*}$ ) $\partial L^{2}\left(q, 1 ; k_{g-1}^{*}\right) / \partial q^{2} \geq \partial L^{2}\left(q, 1 ; k_{g}^{*}\right) / \partial q^{2}, \mathrm{~g}=2, . ., \mathrm{G}$.

Rewrite $\Delta Z$ as $\Delta Z=\sum_{g=1}^{G} \Delta Z_{g}=P 1+P 2+P 3$, where 


$$
\begin{aligned}
P 1= & \alpha_{1 G} \sum_{g=1}^{G} C_{g}(\hat{q})+\left[\alpha_{1(G-1)}-\alpha_{1 G}\right] \sum_{g=1}^{G-1} C_{g}(\hat{q})+\ldots+\left[\alpha_{11}-\alpha_{12}\right] C_{1}(\hat{q}) \\
P 2= & \alpha_{2 G} \sum_{g=1}^{G} B_{2 g}+\left[\alpha_{2(G-1)}-\alpha_{2 G}\right] \sum_{g=1}^{G-1} B_{2 g}+\ldots+\left[\alpha_{21}-\alpha_{22}\right] B_{21} \\
P 3= & -\int_{0}^{\hat{q}}\left[\partial^{2} L\left(q, 1 ; k_{G}^{*}\right) / \partial q^{2}\right] \sum_{g=1}^{G} C_{g}(q) d q \\
& -\int_{0}^{\hat{q}}\left[\partial^{2} L\left(q, 1 ; k_{G-1}^{*}\right) / \partial q^{2}-\partial^{2} L\left(q, 1 ; k_{G}^{*}\right) / \partial q^{2}\right] \sum_{g=1}^{G-1} C_{g}(q) d q \\
& -\ldots-\int_{0}^{\hat{q}}\left[\partial^{2} L\left(q, 1 ; k_{1}^{*}\right) / \partial q^{2}-\partial^{2} L\left(q, 1 ; k_{2}^{*}\right) / \partial q^{2}\right] C_{1}(q) d q
\end{aligned}
$$

Since $\alpha_{1(g-1)} \leq \alpha_{1 g}$, and since from Lemma $3, \sum_{g=1}^{G} C_{g}(\hat{q})=\sum_{g=1}^{G} B_{1 g}=0$, it follows that if condition (A.11.1) holds for $\hat{q}$, then P1 $\geq 0$. Since $\alpha_{2(g-1)} \leq \alpha_{2 g}$ and $\sum_{g=1}^{G} B_{2 g}=0$, it follows that if condition (A.11.2) holds, then P2 $\geq 0$. Finally, since $\partial^{2} L\left(q, 1 ; k_{G}^{*}\right) / \partial q^{2} \geq 0$ and $\partial L^{2}\left(q, 1 ; k_{g-1}^{*}\right) / \partial q^{2} \geq \partial L^{2}\left(q, 1 ; k_{g}^{*}\right) / \partial q^{2}$, it follows that if condition (A.11.1) holds for all $q, 0 \leq q \leq \hat{q}$, then $\mathrm{P} 3 \geq 0$. Then (A.11.1) and (A.11.2) are sufficient for $\Delta \mathrm{Z} \geq 0$.

\section{Necessity}

Assume that condition (A.11) is not satisfied, and consider three cases:

Case 1. There exists a positive integer $h$ and an interval Q such that $\sum_{g=1}^{h} C_{g}(q)>0, q \in Q, h \leq G$.

In this case there exists a set of numbers $\varepsilon_{A g}, \varepsilon_{B g}, \varepsilon_{C g}$, with $\varepsilon_{C g} \geq 0, \mathrm{~g}=1, \ldots, \mathrm{G}$ that are nonincreasing in g such that, $\sum_{g=1}^{G} \varepsilon_{C g} C_{g}(q)>0$ for $q \in Q, \sum_{g=1}^{G} \varepsilon_{A g} C_{g}(\hat{q}) \geq 0$, and $\sum_{g=1}^{G} \varepsilon_{B g} B_{2 g} \geq 0$. This is true because the set of numbers that are non-increasing in $\mathrm{g}$ includes $\varepsilon_{C g}=1, g=1, . ., h ; \varepsilon_{C g}=0, g=h+1, . ., G ; \quad \varepsilon_{A g}=0, g=1, . ., G ; \varepsilon_{B g}=0, g=1, . ., G$.

Case 2. There exists a positive integer h such that $\sum_{g=1}^{h} C_{g}(\hat{q})>0, h \leq G$.

In this case there exists a set of numbers $\varepsilon_{A g}, \varepsilon_{B g}, \varepsilon_{C g}$, with $\varepsilon_{C g} \geq 0, \mathrm{~g}=1, . ., \mathrm{G}$ that are nonincreasing in g such that, $\sum_{g=1}^{G} \varepsilon_{A g} C_{g}(\hat{q})>0, \sum_{g=1}^{G} \varepsilon_{C g} C_{g}(q) \geq 0$ for $q \in Q$, and $\sum_{g=1}^{G} \varepsilon_{B g} B_{2 g} \geq 0$. This is true because the set of numbers that are non-increasing in $\mathrm{g}$ includes $\varepsilon_{A g}=1, g=1, ., h ; \varepsilon_{A g}=0, g=h+1, . ., G ; \quad \varepsilon_{C g}=0, g=1, . ., G ; \varepsilon_{B g}=0, g=1, . ., G$. 
Case 3. There exists a positive integer h such that $\sum_{g=1}^{h} B_{2 g}>0, h \leq G$.

In this case there exists a set of numbers $\varepsilon_{A g}, \varepsilon_{B g}, \varepsilon_{C g}$, with $\varepsilon_{C g} \geq 0, \mathrm{~g}=1, \ldots, \mathrm{G}$ that are nonincreasing in g such that, $\sum_{g=1}^{G} \varepsilon_{B g} B_{2 g}>0, \quad \sum_{g=1}^{G} \varepsilon_{C g} C_{g}(q) \geq 0$ for $q \in Q$, and $\sum_{g=1}^{G} \varepsilon_{A g} C_{g}(\hat{q}) \geq 0$. This is true because the set of numbers that are non-increasing in $\mathrm{g}$ includes $\varepsilon_{B g}=1, g=1, . ., h ; \varepsilon_{B g}=0, g=h+1, . ., G ; \quad \varepsilon_{C g}=0, g=1, . ., G ; \varepsilon_{A g}=0, g=1, . ., G$.

Let $V(.,$.$) be a 3$-smooth function with two arguments that is symmetric and homogeneous of degree one, and let $U(q)=V(q, 1)$, where $U(q)$ has properties, $U(0)=0, \partial U(\hat{q}) / \partial q=0$, and $\partial^{2} U(q) / \partial q^{2}>0, q \in Q$, else $\partial^{2} U(q) / \partial q^{2}=0$. Then in case 1,2 and 3 there exist non-negative numbers, $\left[\partial^{2} U(q) / \partial q^{2}\right] \varepsilon_{C g}$ and numbers $-\varepsilon_{B g}$ and $-\varepsilon_{A g}, g=1, . ., G$ such that, (A.14) $\sum_{g=1}^{G}\left\{-\varepsilon_{B g} B_{2 g}+\left[\varepsilon_{C g} \partial U(\hat{q}) / \partial q-\varepsilon_{A g}\right] C_{g}(\hat{q})-\int_{0}^{\hat{q}} \varepsilon_{C g}\left[\partial^{2} U(q) / \partial q^{2}\right] C_{g}(q) d q\right\}<0$.

Let $L\left(s_{1 j}, s_{2 j} ; k_{j}\right)$ and $\lambda\left(k_{j}\right)$ be functions that not only have the properties of $L()$ and $\lambda()$ in Theorem 1 , but also satisfy,

(A.15) $\partial L\left(\hat{q}, 1 ; k_{g}^{*}\right) / \partial q+\lambda\left(k_{g}^{*}\right)=\varepsilon_{C g} \partial U(\hat{q}) / \partial q-\varepsilon_{A g}$

$$
\lambda\left(k_{g}^{*}\right)=-\varepsilon_{B g}
$$

Note that since $\varepsilon_{B g}$ is non-increasing in $\mathrm{g}$ (and thereby $-\varepsilon_{B g}$ is non-increasing in $k_{g}$ ), $\partial \lambda\left(k_{g}^{*}\right) / \partial k_{g}^{*} \leq 0$, which is consistent with the Theorem 1 properties of $\lambda()$. Since $\varepsilon_{A g}$ is nonincreasing in $\mathrm{g}$ (and thereby $-\varepsilon_{A g}$ is non-increasing in $\left.k_{g}\right), \partial^{2} L\left(\hat{q}, 1 ; k_{g}^{*}\right) / \partial q \partial k_{g}^{*}+\partial \lambda\left(k_{g}^{*}\right) / \partial k_{g}^{*} \leq 0$, which accords with part (c) of Theorem 1, and since $\partial^{2} U(q) / \partial q^{2} \geq 0$ and $\varepsilon_{C g}$ non-negative, $\partial^{2} L\left(q, 1 ; k_{g}\right) / \partial q^{2} \geq 0$, which is consistent with part (e) of Theorem 1 . In addition, since $\varepsilon_{C g}$ is nonincreasing in $\mathrm{g}, \partial^{2} L\left(q, 1 ; k_{g}\right) / \partial q^{2}$ is non-decreasing in $k_{g}$, which is consistent with part (d) of Theorem 1. Finally, (a) and (b) of Theorem 1 are satisfied since $U(0)=0$ and $U(q)=V(q, 1)$, where $V()$ is symmetric and homogeneous of degree one. Then substituting the expressions in (A.15) into (A.14), if condition (A.11) is not satisfied (as it is not in cases 1-3), there exist functions $L($ ) and $\lambda($ ) that conform to the properties of Theorem 1 such that,

$$
\sum_{g=1}^{G}\left\{\lambda\left(k_{g}^{*}\right) B_{2 g}+\left[\partial L\left(\hat{q}, 1 ; k_{g}^{*}\right) / \partial q+\lambda\left(k_{g}^{*}\right)\right] C_{g}(\hat{q})-\int_{0}^{\hat{q}} \partial^{2} L\left(q, 1 ; k_{g}^{*}\right) / \partial q^{2} C_{g}(q) d q\right\}<0 .
$$

Then using (A.12), if condition (A.11) is not satisfied, there exist functions $L()$ and $\lambda($ )such that, $\Delta Z=\sum_{g=1}^{G} \Delta Z_{g}=\sum_{g=1}^{G}\left\{\lambda\left(k_{g}^{*}\right) B_{2 g}+\left[\partial L\left(\hat{q}, 1 ; k_{g}^{*}\right) / \partial q+\lambda\left(k_{g}^{*}\right)\right] C_{g}(\hat{q})-\int_{0}^{\hat{q}} \partial^{2} L\left(q, 1 ; k_{g}^{*}\right) / \partial q^{2} C_{g}(q) d q\right\}<0$

which establishes the claim. 
Proof of Corollary 2.1.

From Lemma 3(b) $C_{g}(\hat{q})=\sum_{j=1}^{T_{g}} s_{1 j g}^{x}-\sum_{j=1}^{T_{g}} s_{1 j g}^{y}=B_{1 g}$. Then from Theorem 2, for all measures of segregation $O\left(\right.$ ) satisfying properties $1-4,5^{\prime}-8^{\prime}$, and for all measures of prestige $k$ satisfying $k_{1}^{*}>k_{2}^{*}>\ldots>k_{G}^{*} \geq 0$ and $k_{j g}=k_{g}^{*}, j=1, . ., T_{g}, g=1, . ., G$, the following condition is necessary for $O(x ; k) \geq O(y ; k): \sum_{g=1}^{\tau} C_{g}(\hat{q})=\sum_{g=1}^{\tau} B_{1 g} \leq 0$, and $\sum_{g=1}^{\tau} B_{2 g} \leq 0, \tau=1, \ldots, G$, which completes the proof. 


\section{Endnotes}

${ }^{1}$ For example, Cutler and Glaeser (1997), Flückiger and Silber (1999), and Reardon, Yun, and Eitle (2000), Weeden (2004), Jenkins, Micklewright, and Schnepf (2006), Frankel and Volij (2011)

2 See the discussion in Nakao and Treas (1994).

3 A large literature on sequential dominance and equivalence scales followed Atkinson and Bourguignon (1987). For example, see Jenkins and Lambert (1993), Chambaz and Maurin (1998), Bourguignon (1989), Ok and Lambert (1999), and Fluerbaey, Hagnere, and Trannoy (2003).

${ }^{4}$ As such, the term "economic status" is defined so that a group's economic status is not affected by changes in the gender, ethnic, or racial composition of the group.

${ }_{5}^{5}$ See Duncan and Duncan (1955) and Hutchens (1991).

${ }^{6}$ An $x$ matrix with non-integer values is plausible. Part-time workers could be treated as fractional workers or as irrational numbers (for example, while a full-time worker is counted as '1,' a part-time worker is counted as the square root of .3).

7 There remains a good question of whether the arguments in this paper can be extended to a measure of inequality for more than two types of people (e.g., more than two ethnic groups) such that the $x$ matrix in $\mathrm{O}(x ; k)$ has more than two rows. That, however, will have to be a topic for future work.

8 In particular, an index $\mathrm{O}(\mathrm{x})$ satisfies the seven properties if and only if $O(x)=G\left\{\sum_{i=1}^{T} H\left(x_{1 i} / N_{1}, x_{2 i} / N_{2}\right)\right\}$, where

$G\{\}$ is a continuous and strictly increasing function, and $H()$ is a continuous, symmetric, and strictly convex function that is homogeneous of degree one with $H(0,0)=0$.

9 Property P7 differs slightly from the movement between groups property in Hutchens (2001). The earlier treatment assumed strict inequality. In order to be consistent with the index in Theorem 1, P7 assumes $O\left(x ; k^{0}\right) \leq O\left(y ; k^{0}\right)$.

10 This could be argued more formally by asserting that a measure of segregation should be weakly separable in any partition of the T occupations into mutually exclusive and exhaustive subsets. By Gorman's overlapping Theorem (Gorman, 1968), this implies that the measure should be additive.

11 By implication, P7 deals with a disequalizing movement with unchanged economic status, since in P7 all groups have the same status $\left(k=k^{0}\right)$.

12 The second inequality in $\mathrm{P}^{\prime}$ '(b) can be derived from P7'(a). Given that $\mathrm{O}(x ; k)$ is continuous over its domain, it can be shown that as $x_{2 f}=x_{2 g}=x_{2 h}$ approaches zero, P7'(a) implies $O(y ; k) \leq O(z ; k)$.

13 It is important to be clear about what P8' does not say. It does not say that segregation among high economic status occupations is worse than segregation among low economic status occupations. For example, P8' neither says nor implies that measured segregation is increasing in $k$ (e.g., increasing in $k_{\mathrm{q}}=k_{\mathrm{r}}$ ). P8' is not a statement about the level of the index, but rather how the index changes in response to a disequalizing transfer.

14 Since the augmented square root index is an additive decomposable measure of segregation, it permits a "within" and "between" decomposition. The within component takes the form, $\Sigma_{\mathrm{g}} w_{\mathrm{g}} O\left(x^{\mathrm{g}}\right)$, where $O\left(x^{g}\right)=1-\sum_{j=1}^{T_{g}}\left(s_{1 j g} s_{2 j g}\right)^{1 / 2}$, and $\mathrm{w}_{\mathrm{g}}=\left(\gamma\left(k_{\mathrm{g}}\right) / \gamma(\tilde{k})\right)\left(\mathrm{N}_{1}\left(x^{\mathrm{g}}\right) / \mathrm{N}_{1}(x)\right)^{1 / 2}\left(\mathrm{~N}_{2}\left(x^{\mathrm{g}}\right) / \mathrm{N}_{2}(x)\right)^{1 / 2}$, while the between component takes the form, $\mathrm{B}=b-\gamma(\tilde{k}) 2 \lambda(\tilde{k})+\gamma(\tilde{k})\left(\Sigma_{\mathrm{g}}\left[\left(-\mathrm{b} / \gamma\left(\mathrm{k}_{\mathrm{g}}\right)\right)\left(\mathrm{N}_{1}\left(x^{\mathrm{g}}\right) / \mathrm{N}_{1}(x)\right)^{1 / 2}\left(\mathrm{~N}_{2}\left(x^{\mathrm{g}}\right) / \mathrm{N}_{2}(x)\right)^{1 / 2}+\lambda\left(\mathrm{k}_{\mathrm{g}}\right)\left(\mathrm{N}_{1}\left(x^{\mathrm{g}}\right) / \mathrm{N}_{1}(x)+\mathrm{N}_{2}\left(x^{\mathrm{g}}\right) / \mathrm{N}_{2}(x)\right)\right]\right.$. For example, one could use the augmented square root index to analyze intertemporal changes in (a) segregation between subsets (changes in B, (b) segregation within subsets (changes in $\Sigma_{\mathrm{g}} \mathrm{g}_{\mathrm{g}} O\left(x^{\mathrm{g}}\right)$ )), and (c) changes within each of the subsets (the $O\left(x^{\mathrm{g}}\right), \mathrm{g}=1, \mathrm{G}$ ). Note that $O\left(x^{\mathrm{g}}\right)$, is not augmented - it is a standard square root index (same $k_{\mathrm{g}}$ ). 
15 To see this, consider equation (6). With $s_{2 \mathrm{f}}=s_{2 \mathrm{~h}}=0$, the final term in (6), $-\frac{b}{\gamma\left(k_{j}\right)} \sqrt{s_{1 j} s_{2 j}}$, equals zero for $\mathrm{j}=\mathrm{f}, \mathrm{h}$. Thus, the P7'(b) movement of $d$ people from high status group f to lower status group h changes the equation (6) index by $\gamma(\tilde{k})\left[\lambda\left(k_{f}\right)\left(-d / N_{1}\right)+\lambda\left(k_{h}\right)\left(d / N_{1}\right)\right]$. With $\gamma(\tilde{k})>0, k_{\mathrm{f}}>k_{\mathrm{h}}$, and $\lambda\left(k_{\mathrm{j}}\right)$ nonincreasing in $k_{\mathrm{j}}$, the resulting change in the equation (6) index is non-negative. Thus, due to the term $\sum_{j=1}^{T}\left[\lambda\left(k_{j}\right)\left(s_{1 j}+s_{2 j}\right)\right]$, the equation (6) index behaves precisely as required by $\mathrm{P} 7^{\prime}(\mathrm{b})$.

16 See derivation of equation A.13 in the proof of Theorem 2 in the appendix.

17 It is not easy to provide intuition for Theorem 2, but one way to think about it is to suppose that 9.1 is satisfied and 9.2 is violated for $\tau=\tau^{*}$, i.e., $\sum_{g=1}^{\tau^{*}} B_{2 g}>0$. Then according to Theorem 2 , there can exist an index that both satisfies the conditions of Theorem 1 AND yields $\mathrm{O}(x ; k)<\mathrm{O}(y ; k)$. Consider then the index that results when $\lambda\left(k_{1}^{*}\right)=\lambda\left(k_{2}^{*}\right)=\ldots=\lambda\left(k_{\tau^{*}}^{*}\right)<0 ; \quad \lambda\left(k_{\tau^{*}+1}^{*}\right)=\lambda\left(k_{\tau^{*}+2}^{*}\right)=\ldots=\lambda\left(k_{G}^{*}\right)=0 ; C_{g}(\hat{q})=0, g=1, G ;$ and $\partial^{2} L\left(q, 1 ; k_{g}^{*}\right) / \partial q^{2}=0,0 \leq q \leq \hat{q}, \mathrm{~g}=$ $1, \ldots, \mathrm{G}$. Since $\Delta Z=\sum_{g=1}^{G} \Delta Z_{g}=\sum_{g=1}^{G}\left\{\alpha_{1 g} C_{g}(\hat{q})+\alpha_{2 g} B_{2 g}-\int_{0}^{\hat{q}}\left[\partial^{2} L\left(q, 1 ; k_{g}^{*}\right) / \partial q^{2}\right] C_{g}(q) d q\right\}$ where $\alpha_{1 g}=\lambda\left(k_{g}^{*}\right)+\partial L\left(\hat{q}, 1 ; k_{g}^{*}\right) / \partial q$, and $\alpha_{2 g}=\lambda\left(k_{g}^{*}\right)$, in this case $\Delta \mathrm{Z}<0$. Then for this violation of 9.2, there does, indeed, exist an index that satisfies the conditions of Theorem 1 and yields $\mathrm{O}(x ; k)<\mathrm{O}(y ; k)$. The same approach applies to 9.1, i.e., suppose 9.1 is violated for $\tau=\tau^{*}$ and $q=q^{*}$. Then with $\lambda\left(k_{\mathrm{g}}\right)=0, \mathrm{~g}=1, \ldots, \mathrm{G}$, there can exist a function $L()$ that satisfies the conditions of Theorem 1 and yields $\Delta \mathrm{Z}<0$.

18 An alternative way to think about Theorem 2 and its corollary is to note that the corollary states a kind of firstdegree dominance condition while Theorem 2 states a kind of second-degree dominance condition. This is essentially the approach taken in Atkinson and Bourguignon (1987). That approach is not taken here because the "first-degree dominance condition" does not utilize all of the properties of the Theorem 1 index, and the condition is only useful in the context of examining whether the data satisfy the Theorem 2 criteria.

19 See James Foster, Jack Greer, and ErikThorbecke (1984).

20 There is a good discussion of this in Sen (1997), pages 168-188.

21 Parameters are specified as $\alpha_{\gamma}=1, \beta_{\gamma}=.888889, \alpha_{\lambda}=0, \beta_{\lambda}=.36, b=1.8$, with $\operatorname{med}\left(\hat{k}_{j}\right)=.5$, and $\tilde{k}=\max \left(\hat{k}_{j}\right)$ $=1$, implying $\lambda(\tilde{k})=\lambda(1)$ and $\gamma(\tilde{k})=\gamma(1)$. This parameterization of the index in (6) is derived from six equations. First, let the minimum value of the index be zero. The minimum occurs when all type 1 and type 2 people are in completely integrated occupations $\left(s_{1 \mathrm{j}}=s_{2 \mathrm{j}}\right)$, all of which have the highest status level. In that case,

$$
O(x ; k)=0=b-\gamma(\tilde{k}) 2 \lambda(\tilde{k})+\gamma(\tilde{k})\left[2 \lambda\left(\max \left(\hat{k}_{j}\right)\right)-\frac{b}{\gamma\left(\max \left(\hat{k}_{j}\right)\right)}\right]
$$

Second, let $\mu$ be the maximum value of the index, a parameter selected by the researcher. The maximum occurs when all type 1 and type 2 people are in completely segregated occupations (if $s_{1 j}>0$, then $s_{2 j}=0$ ), and all of those occupations have the lowest status level. In that case,

(2) $O(x ; k)=\mu=b-\gamma(\tilde{k}) 2 \lambda(\tilde{k})+\gamma(\tilde{k}) 2 \lambda\left(\min \left(\hat{k}_{j}\right)\right)$

Next, when all occupations have the median status level $\left(\operatorname{med}\left(\hat{k}_{j}\right)\right)$, let the index equal the status-free square root index plus 1 . Since the square root index lies between 0 and 1 , it follows that at the median status level, this augmented index lies between 1 and 2 . Then when all type 1 and type 2 people are in completely integrated occupations and all of those occupations have the median status level, 


$$
O(x ; k)=1=b-\gamma(\tilde{k}) 2 \lambda(\tilde{k})+\gamma(\tilde{k})\left[2 \lambda\left(\operatorname{med}\left(\hat{k}_{j}\right)\right)-\frac{b}{\gamma\left(\operatorname{med}\left(\hat{k}_{j}\right)\right)}\right]
$$

and when all type 1 and type 2 people are in completely segregated occupations with all of those occupations at the median status level,

(4) $O(x ; k)=2=b-\gamma(\tilde{k}) 2 \lambda(\tilde{k})+\gamma(\tilde{k}) 2 \lambda\left(\operatorname{med}\left(\hat{k}_{j}\right)\right)$

Finally, as noted in the text, the functions for $\gamma$ and $\lambda$ are specified as

(5) $\gamma\left(\hat{k}_{j}\right)=\alpha_{\gamma}-\beta_{\gamma}\left(\hat{k}_{j}\right)$ and (6) $\lambda\left(\hat{k}_{j}\right)=\alpha_{\lambda}-\beta_{\lambda}\left(\hat{k}_{j}\right)$

By assumption $\alpha_{\gamma}=1$ and $\alpha_{\lambda}=0$. A solution to (1) occurs at $\tilde{k}=\max \left(\hat{k}_{j}\right)$, so set $\tilde{k}=\max \left(\hat{k}_{j}\right)$. Given the

researcher's choice of $\mu$, and the observed value of $\operatorname{med}\left(\hat{k}_{j}\right)$ in the data, the remaining five equations can be solved

for the three unknown parameters $\left(\beta_{\gamma}, \beta_{\lambda}, b\right)$. In particular, for $\mu=2.2$ and $\operatorname{med}\left(\hat{k}_{j}\right)=.50, \beta_{\gamma}=.88889, \beta_{\lambda}=.36$, and $b=1.8$.

22 Useful reviews of (and contributions to) this literature may be found in Mary King (1992), Fluckiger and Silber (1999), and the bibliography in Cotter, Hermsen, and Vanneman (2005)

${ }^{23}$ They recoded occupations to create a 179 occupation scheme; correspondence with these authors indicates that the recodes have subsequently been lost. The PUMS 389 occupation scheme yields, however, roughly similar results. In particular, the rankings of the dissimilarity indexes in this paper's Tables 2 and 3 are identical to those in Cotter, Hermsen, and Vanneman (2005).

24 See Hauser and Warren (1997) for a survey and critique.

25 See Nam and Boyd (2004) for a description of how the index is constructed. See http://usa.ipums.org/usa/chapter4/chapter4.shtml, for a discussion of how Michigan researchers applied the index to the Census PUMS. The index ranges from 1 (e.g., dishwasher) to 100 (e.g., physician or surgeon).

26 The five categories are $k_{1}=5$ (high status) if $100>\mathrm{NPB}$ index $>80 ; k_{2}=4$ if $80>\mathrm{NPB}$ index $>60, \ldots, k_{5}=1$ (low status ) if $20>$ NPB index $>0$. This translates into the values of $\hat{k}_{j}$ in the text. Of course Augmented Index A could also be computed using each and every value of the NPB index. When this was done, the column 6 ordinal results in Table 2 and 3 were unchanged. The five category scheme is used here because it accords well with the subsequent examination of Theorem 2, and because the goal is to apply the measures introduced here to data and thereby assess their utility.

27 See Cotter, Hermsen, and Vanneman (2005), Table 8.

28 More precisely, there are two reasons for the difference. First, the way that a demographic group is distributed over the hierarchy can affect a status-augmented measure without similarly affecting a status-free measure. To illustrate, on average Asians are in higher status occupations than Hispanics. While that fact has no effect on a status-free measure, it can cause differences in a status-augmented measure; even if Asians and Hispanics had the same status-free measure, the fact that Asians experience that status-free segregation in higher status occupations results in a smaller status-augmented measure for Asians. Second, the way that segregation is distributed over the hierarchy can affect a status-augmented measure without similarly affecting a status-free measure. For example, suppose Hispanics and African Americans have the same status-free measure of segregation and are equally represented (same percentages) at each level of the hierarchy. Suppose, however that in high status occupations the segregation experienced by African Americans tends to be greater than that experienced by Hispanics, while the opposite is true in low status occupations. Then despite the fact that the status-free measures are the same, the augmented square root index will be larger for African Americans.

Certainly a goal of future work should be to develop a decomposition that captures these two reasons for differences between status-free and status-augmented measures. For present purposes, however, it is sufficient to note that status-free and status-augmented measures paint different pictures of segregation. 
${ }^{29}$ For the pair of race/ethnic groups being examined, $\hat{q}$ is the maximum value of $s_{1 \mathrm{j}} / s_{2 \mathrm{j}}$ in occupations with $s_{2 \mathrm{j}}>0$, $\mathrm{j}=1, . ., \mathrm{T}$. For example, for Hispanics and African Americans $\hat{q}=69.903786$.

${ }^{30}$ I am indebted to Serif Aziz Simsir for checking and improving the proofs in this appendix, and to Matt Sweeney and Phil Armour for programming help. 
Reference List

Atkinson, Anthony B., and Francois Bourguignon. "Income Distribution and Differences in Needs." in Arrow and the Foundation of the Theory of Economic Policy, ed., G. F. Feiwel, 350-370. London: Macmillan, 1987.

Berge, Claude. Topological Spaces. Mineola, New York : Dover, 1997.

Bourguignon, Francois. "Family Size and Social Utility: Income Distribution Dominance Criteria." Journal of Econometrics 42 (1989): 67-80.

Chambaz, Christine, and Eric Maurin. "Atkinson and Bourguignon's Dominance Criteria: Extended and Applied to the Measurement of Poverty in France." Review of Income and Wealth 44, no. 4 (1998): 497-513.

Cotter, David A., Joan M. Hermsen, and Reeve Vanneman. "Gender Inequality at Work." in The American People: Census 2000. eds. Reynolds Farley, and John Haaga, 107-38. Russell Sage Foundation and the Population Reference Bureau, 2005.

Cutler, David M., and Edward L. Glaeser. "Are Ghettos Good or Bad?" Quarterly Journal of Economics 112, no. 3 (1997): 827-72.

Duncan, Otis D., and Beverly Duncan. "A Methodological Analysis of Segregation Indices." American Sociological Review 20 (1955): 210-217.

Fleurbaey, Marc, Cyrille Hagneré, and Alain Trannoy. "Welfare Comparisons With Bounded Equivalence Scales." Journal of Economic Theory 110 (2003): 309-36.

Foster, James, Jack Greer, and Erik Thorbecke. "A Class of Decomposable Poverty Measures." Econometrica 52, no. 3 (1984): 761-66.

Foster, James E. "Normative Measurement: Is Theory Relevant?" American Economic Review 84, no. 2 (1994): pp. 365-70.

Glass Ceiling Commission. Report of the Glass Ceiling Commission, U.S. Department of Labor, Washington, DC, 1995.

Gorman, William M. "The Structure of Utility Functions." Review of Economic Studies 35 (1968): $367-90$.

Hauser, Robert M., and John Robert Warren. "Socioeconomic Indexes for Occupations: A Review, Update, and Critique." Sociological Methodology 27 (1997): 177-298.

Hutchens, Robert M. "Numerical Measures of Segregation: Desirable Properties and Their Implications." Mathematical Social Sciences 42, no. 1 (2001): 13-29. 
. "One Measure of Segregation." International Economic Review 45, no. 2 (2004): 555-77.

. "Segregation Curves, Lorenz Curves, and Inequality in the Distribution of People Across Occupations." Mathematical Social Sciences 21 (1991): 31-51.

Jenkins, Stephen P., and Peter J. Lambert. "Ranking Income Distributions When Needs Differ." Review of Income and Wealth 39, no. 4 (1993): 337-56.

King, Mary C. "Occupational Segregation by Race and Sex, 1940 - 1988." Monthly Labor Review 115 (1992): 30-37.

Nakao, Keiko, and Judith Treas. "Updating Occupational Prestige and Socioeconomic Scores: How the New Measures Measure Up." ed. Peter Marsden. Sociology Methodology (1994).

Ok, Efe A., and Peter J. Lambert. "On Evaluating Social Welfare by Sequential Generalized Lorenz Dominance." Economics Letters 63 (1999): 45-53.

Reardon, Sean F., John T. Yun, and Tamela M. Eitle. "The Changing Structure of School Segregation: Measurement and Evidence of Multiracial Metropolitan-Area School Segregation, 1989-1995." Demography 37, no. 3 (2000): 351-64.

Sen, Amartya. On Economic Inequality. Enlarged edition with an annex by James Foster and Amartya Sen. Oxford: Clarendon Press, 1997. 